\title{
Molecular gas content and SFR in Hickson compact groups: enhanced or deficient? ${ }^{\star}$
}

\author{
V. Martinez-Badenes ${ }^{1}$, U. Lisenfeld $^{2}$, D. Espada ${ }^{1,3}$, L. Verdes-Montenegro $^{1}$, S. García-Burillo $^{4}$, \\ S. Leon ${ }^{5}$, J. Sulentic ${ }^{1}$, and M. S. Yun ${ }^{6}$ \\ ${ }^{1}$ Instituto de Astrofísica de Andalucía (IAA/CSIC), Apdo. 3004, 18080 Granada, Spain \\ e-mail: vicentm@iaa.es \\ 2 Departmento de Física Teórica y del Cosmos, Facultad de Ciencias, Universidad de Granada, Spain \\ e-mail: ute@ugr.es \\ 3 National Astronomical Observatory of Japan, 2-21-1, Osawa, Mitaka, 181-8588 Tokyo, Japan \\ ${ }^{4}$ Observatorio Astronómico Nacional (OAN) Observatorio de Madrid, C/Alfonso XII 3, 28014 Madrid, Spain \\ 5 Joint ALMA Observatory/ESO, Vitacura, Santiago, Chile \\ ${ }^{6}$ Department of Astronomy, University of Massachusetts, Amherst, MA 01003, USA
}

Received 17 May 2011 / Accepted 24 January 2012

\begin{abstract}
Aims. We study the effect of the extreme environment in Hickson compact groups (HCGs) on the molecular gas mass, $M_{\mathrm{H}_{2}}$, and the star formation rate (SFR) of galaxies as a function of atomic hydrogen (HI) content and evolutionary phase of the group.

Methods. We selected a redshift-limited $(D<100 \mathrm{Mpc}$ ) sample of 88 galaxies in 20 HCGs with available atomic hydrogen (HI) VLA maps, covering a wide range of $\mathrm{HI}$ deficiencies and evolutionary phases of the groups and containing at least one spiral galaxy. We observed the $\mathrm{CO}(1-0)$ and $\mathrm{CO}(2-1)$ lines with the IRAM $30 \mathrm{~m}$ telescope for 47 galaxies. Together with literature data, our sample contains $\mathrm{CO}(1-0)$ spectra for 86 galaxies. We derived the far-infrared (FIR) luminosity $\left(L_{\mathrm{FIR}}\right)$ from IRAS data and used it as a tracer of the SFR. We calculated the HI mass $\left(M_{\mathrm{HI}}\right), L_{\mathrm{FIR}}$, and $M_{\mathrm{H}_{2}}$ deficiencies, based on the values expected from $L_{B}$ and $L_{K}$ in isolated galaxies from the AMIGA sample. We limited our statistical analysis to spiral galaxies, since the large number of upper limits did not allow drawing strong conclusions about $M_{\mathrm{H}_{2}}$ and $L_{\mathrm{FIR}}$ in early-type galaxies.

Results. The mean deficiencies of $L_{\mathrm{FIR}}$ and $M_{\mathrm{H}_{2}}$ of spiral galaxies in HCGs are close to 0, indicating that their average SFR and molecular gas content are similar to those of isolated galaxies. However, there are indications of an excess of $M_{\mathrm{H}_{2}}(\sim 50 \%)$ in spiral galaxies in HCGs, which can be interpreted, assuming that there is no systematic difference in the CO-to- $\mathrm{H}_{2}$ conversion factor, as either an enhanced molecular gas content or as a higher concentration of the molecular component towards the center in comparison to galaxies in lower density environments. In contrast, the mean $M_{\mathrm{HI}}$ of spiral galaxies in HCGs is only $12 \%$ of the expected value. The specific SFR (sSFR = SFR/stellar mass) tends to be lower for galaxies with higher $M_{\mathrm{H}_{2}}$ or $M_{\mathrm{HI}}$ deficiency. This trend is not seen for the star formation efficiency $\left(\mathrm{SFE}=\mathrm{SFR} / M_{\mathrm{H}_{2}}\right.$ ), which is very similar to isolated galaxies. We found tentative indications of an enhancement of $M_{\mathrm{H}_{2}}$ in spiral galaxies in HCGs in an early evolutionary phase and a decrease in later phases. We suggest that this might be due to an enhancement of the conversion from atomic to molecular gas due to ongoing tidal interactions in an early evolutionary phase, followed by HI stripping and a decrease in the molecular gas content because of lack of replenishment.

Conclusions. The properties of $M_{\mathrm{H}_{2}}$ and $L_{\mathrm{FIR}}$ in galaxies in HCGs are surprisingly similar to those of isolated galaxies, in spite of the much higher $\operatorname{Def}\left(M_{\mathrm{HI}}\right)$ of the former. The trends of the $\mathrm{sSFR}$ and $\operatorname{Def}\left(M_{\mathrm{H}_{2}}\right)$ with $\operatorname{Def}\left(M_{\mathrm{HI}}\right)$ and the evolutionary state indicate, however, that the ongoing interaction might have some effect on the molecular gas and SF.
\end{abstract}

Key words. ISM: molecules - galaxies: evolution - galaxies: ISM - galaxies: interactions - galaxies: star formation galaxies: groups: general

\section{Introduction}

Hickson compact groups (HCGs) (Hickson 1982) are dense and relatively isolated groups of four to eight galaxies in the nearby universe. The combination of high galaxy density (Hickson 1982) and low-density environment coupled with low systemic velocity dispersions $\left(\langle\sigma\rangle=200 \mathrm{~km} \mathrm{~s}^{-1}\right.$, Hickson et al. 1992) make HCGs especially interesting systems for studying how gas content and star formation activity in galaxies are influenced by the environment.

* Full Tables $1-3$, and 5 are only available in electronic form at the CDS via anonymous ftp to

cdsarc.u-strasbg.fr $(130.79 .128 .5)$ or via

http://cdsarc.u-strasbg.fr/viz-bin/qcat?]/A+A/540/A96
The most remarkable effect of multiple and strong interactions between galaxies in HCGs involves an atomic gas (HI) deficiency. VLA measures of individual spiral galaxies in HCGs show they only have $24 \%$ of the atomic hydrogen (HI) mass, $M_{\mathrm{HI}}$, expected from their optical luminosities and morphological types (Verdes-Montenegro et al. 2001). The inferred deficiency becomes even greater if one assumes that many of the lenticular galaxies, which are overrepresented in HCGs, are stripped spirals. Verdes-Montenegro et al. (2001) propose an evolutionary sequence for HCGs in which the HI is continuously removed from the galaxies, finally leading to groups where most of the HI is located outside of the galaxies. However, not only are the individual galaxies in HCGs HI deficient, but also HCGs are so as a whole (Verdes-Montenegro et al. 2001). This leads to the still 
open question of where the missing HI has gone and by which mechanism it was removed. To investigate the role played by a hot intragroup medium (IGM), Rasmussen et al. (2008) performed Chandra and XMM-Newton observations to study eight of the most HI deficient HCGs. They find bright X-ray emission in only four groups suggesting that galaxy-IGM interactions are not the dominant mechanism driving cold gas out of the galaxies. Borthakur et al. (2010) find with new single-dish Green Bank Telescope (GBT) observations of HGCs an important diffuse, low-column density intragroup HI component, missed by VLA observations. Taking these components into account reduced, but did not completely eliminate, the HI-deficiency of the groups.

The effect of an extreme environment on molecular gas properties is controversial. An enhancement of molecular gas content with respect to isolated galaxies has been reported for strongly interacting systems (Casasola et al. 2004, and references therein), defined in that work as galaxies appearing to be clearly interacting with nearby objects, presenting tidal tails or bridges, and merging systems and galaxies with disturbed structures. With respect to galaxies in clusters, no deficiency in the molecular gas content has been found in any global studies of the Virgo cluster (Kenney \& Young 1986; Boselli et al. 2002) and the Coma Supercluster (Casoli et al. 1991; Boselli et al. 1997) in spite of the large HI deficiencies that some galaxies presented. The spatially resolved study of Fumagalli et al. (2009) found, however, that a significant number $(\sim 40 \%)$ of HI-deficient spiral galaxies were also depleted in molecular gas, if the HI was removed from within the optical disk. Scott et al. (in prep.) find a trend for spirals in Abell 1367 in more evolved evolutionary states to be more depleted in $M_{\mathrm{H}_{2}}$ than those in less evolved evolutionary states. Thus, there are indications that the cluster environment does affect the molecular gas content.

Observations of the molecular gas in galaxies in HCGs have given until now contradictory results. Leon et al. (1998) found the $M_{\mathrm{H}_{2}} / L_{B}$ ratio of galaxies in HCGs to be enhanced with respect to a sample of field and interacting galaxies. In contrast, Verdes-Montenegro et al. (1998) found no evidence for an enhancement of the molecular gas mass $\left(M_{\mathrm{H}_{2}}\right)$ in $\mathrm{HCG}$ galaxies relative to a sample of isolated galaxies. Studying the relation between atomic and molecular gas for a sample of 32 spiral galaxies, Verdes-Montenegro et al. (2001) have found tentative evidence of a depressed molecular gas content in HI deficient galaxies in HCGs.

The level of star formation (SF) in HCGs has also been subject to considerable debate with original claims of a far-infrared (FIR) excess (Hickson et al. 1989), which was subsequently challenged (Sulentic \& de Mello Rabaca 1993). From the enhanced SF observed in galaxy pairs (Xu \& Sulentic 1991), an increase in SF in HCGs might be expected as a consequence of the continuous encounters and tidal interactions that take place within such groups. Nevertheless, the star formation rate (SFR) in HCGs, obtained from FIR (Verdes-Montenegro et al. 1998), mid-infrared (Bitsakis et al. 2010), a combination of midinfrared and ultraviolet (Tzanavaris et al. 2010), and $\mathrm{H} \alpha$ luminosities (Iglesias-Páramo \& Vílchez 1999) has been found to be similar to those of the control samples.

There have been a few attempts to study the relation of $M_{\mathrm{H}_{2}}$ and $L_{\mathrm{FIR}}$ with the HI properties of the HCG galaxies. Based on $\mathrm{CO}$, FIR, and HI single-dish data, together with VLA maps for eight groups, Verdes-Montenegro et al. (2007) found that the $M_{\mathrm{H}_{2}}$ and $L_{\mathrm{FIR}}$ are lower than expected for HI deficient galaxies, when compared to a well-defined sample of isolated galaxies (AMIGA project, Analysis of the interstellar Medium of Isolated
GAlaxies, http: //amiga.iaa.es; Verdes-Montenegro et al. 2005). A possible explanation for this trend is that, since HI is needed to replenish the molecular clouds and molecular gas is necessary to fuel SF, an HI deficiency can ultimately lead to a decrease in the SFR. However, the result of Verdes-Montenegro et al. (2007) was based on a small sample of galaxies that does not cover the wide range of properties of HCGs and was therefore not statistically significant. On the other hand, while previous works studying $M_{\mathrm{H}_{2}}$ and SFR of galaxies in HCGs (VerdesMontenegro et al. 1998; Leon et al. 1998) were based on larger samples, those did not have the HI mass of the individual galaxies to compare with $M_{\mathrm{H}_{2}}$ and $L_{\mathrm{FIR}}$. Thus, to date, no study of the relation between $M_{\mathrm{H}_{2}}, M_{\mathrm{HI}}$ and the SFR for a statistically significant sample has been carried out.

To shed light on the relations between $M_{\mathrm{H}_{2}}$ and SFR with $M_{\mathrm{HI}}$ properties of the HCGs, we present here a systematic study of galaxies in a sample of 20 HCGs for which we have HI measurements for the entire groups, as well as for a large fraction of the individual galaxies. This enables us to consider $M_{\mathrm{HI}}$ of the galaxies as an additional parameter, as well as the evolutionary phase of the group according to Verdes-Montenegro et al. (2001) (see Sect. 2). We compare the properties of galaxies in HCGs with those of isolated galaxies in the AMIGA sample (VerdesMontenegro et al. 2005). Our goal is to determine whether deviations in the $\mathrm{HI}$ content with respect to isolated galaxies translate into anomalies in the $M_{\mathrm{H}_{2}}$ and the SFR.

The outline of this paper is as follows. We present the sample in Sect. 2. $\mathrm{CO}(1-0)$ and $\mathrm{CO}(2-1)$ data coming either from our observations or from the literature, together with reprocessed IRAS FIR data, are presented in Sect. 3. In Sect. 4 we compare the $M_{\mathrm{H}_{2}}, L_{\mathrm{FIR}}$ (as a tracer of the SFR), and $M_{\mathrm{HI}}$ of the galaxies, studying their deficiencies and their relation with the HI content and evolutionary phase of the group. A discussion of a possible evolutionary sequence for the molecular gas content in the HCGs is presented in Sect. 5. Finally, the conclusions of our work are summarized in Sect. 6.

\section{The samples}

\subsection{Galaxies in HCGs}

Our sample was selected from the revision of the original Hickson (1982) catalog performed by Hickson et al. (1992). From the groups included in that work, we studied 86 galaxies belonging to 20 different HCGs: 7, 10, 15, 16, 23, 25, 30, 31, 37, $40,44,58,67,68,79,88,92,93,97$, and 100 . The groups, which cover all evolutionary stages and a wide range of HI deficiencies, satisfy the following criteria:

- have at least four members, so triplets are excluded, according to the original Hickson (1982) criterion. We also exclude false groups, where a single knotty irregular galaxy has been confused with separated galaxies (Verdes-Montenegro et al. 2001);

- contain at least one spiral galaxy, since we are mainly interested in studying the relation between the SF process and $M_{\mathrm{H}_{2}}$, which are most clearly linked for spiral galaxies;

- are at a distance $D \leq 100 \mathrm{Mpc}$ (assuming $H_{0}=$ $75 \mathrm{~km} \mathrm{~s}^{-1} \mathrm{Mpc}^{-1}$ ), in order to guarantee a good sensitivity and to minimize possible source confusion within the telescope beam. At $100 \mathrm{Mpc}$, the $30 \mathrm{~m}$ beam would have a size of $10.7 \mathrm{kpc}$ and the VLA beam (considering a size of $50^{\prime \prime} \times 50^{\prime \prime}$ ) $24.2 \mathrm{kpc}$. 
Table 1. Basic parameters of the galaxies in the HCG sample.

\begin{tabular}{lccccccccc}
\hline \hline Galaxy & $\begin{array}{c}V \\
\left(\mathrm{~km} \mathrm{~s}^{-1}\right)\end{array}$ & $\begin{array}{c}\sigma_{V} \\
\left(\mathrm{~km} \mathrm{~s}^{-1}\right)\end{array}$ & $\begin{array}{c}D \\
(\mathrm{Mpc})\end{array}$ & $T(\mathrm{RC} 3)$ & $\begin{array}{c}D_{25} \\
(\operatorname{arcmin})\end{array}$ & $\begin{array}{c}B_{\mathrm{c}}^{\mathrm{T}} \\
(\mathrm{mag})\end{array}$ & $\begin{array}{c}\log \left(L_{B}\right) \\
\left(L_{\odot}\right)\end{array}$ & $\begin{array}{c}\log \left(L_{K}\right) \\
\left(L_{\odot}\right)\end{array}$ & $\begin{array}{c}\log \left(M_{\mathrm{HI}}\right) \\
\left(M_{\odot}\right)\end{array}$ \\
$(1)$ & $(2)$ & $(3)$ & $(4)$ & $(5)$ & $(6)$ & $(7)$ & $(8)$ & $(9)$ & $(10)$ \\
\hline $7 \mathrm{a}$ & 4141 & 117 & 53.4 & 1.0 & 2.06 & 12.96 & 10.22 & 11.09 & 9.12 \\
$7 \mathrm{~b}$ & 4175 & 117 & 53.4 & -1.9 & 1.27 & 14.29 & 9.69 & 10.78 & $<7.83$ \\
$7 \mathrm{c}$ & 4347 & 117 & 53.4 & 5.0 & 1.71 & 13.36 & 10.06 & 10.80 & 9.56 \\
$7 \mathrm{~d}$ & 4083 & 117 & 53.4 & -1.4 & 0.94 & 14.04 & 9.79 & 10.11 & 9.00 \\
$10 \mathrm{a}$ & 5104 & 269 & 65.6 & 3.1 & 2.92 & 12.53 & 10.57 & 11.27 & - \\
$\ldots$. & $\ldots$ & $\ldots$ & $\ldots$. & $\ldots$ & $\ldots$. & $\ldots$. & $\ldots$. & $\ldots$. & $\ldots$ \\
\hline
\end{tabular}

Notes. The full table is available in electronic form at the CDS and from http://amiga.iaa.es.

The HCGs in our sample cover the full range of HI contents. Their deviation from normalcy is measured with respect to that of isolated galaxies, as given by Haynes \& Giovanelli (1984). This deviation is usually referred to as deficiency and is defined as the decimal logarithm of the ratio between the sum of the expected HI masses for all the galaxies in the group based on their optical luminosity and morphology and of the HI mass of the entire group as derived from the single-dish observations in Borthakur et al. (2010; see Verdes-Montenegro et al. 2001, and also Sect. 4.2). As a function of their total HI deficiency, the HCGs in our sample can be classified as:

- HCGs with a normal HI content (at least 2/3 of its expected value): HCG 23, 25, 68, and 79;

- HCGs with a slight HI deficiency (between $2 / 3$ and $1 / 3$ of the expected value): HCG 7, 10, 15, 16, 31, 37, 40, 58, 88, 92, 97, and 100;

- HCGs with a large HI deficiency (under $1 / 3$ of the expected value): $\mathrm{HCG} 30,44,67$, and 93.

Verdes-Montenegro et al. (2001) propose an evolutionary sequence model where the HI is continuously stripped from the galaxies. According to this model, HCGs can be classified into three phases as follows. In Phase 1 the $\mathrm{HI}$ is mainly found in the disks of galaxies. In Phase 2, 30\% to $60 \%$ of the HI has been removed from the disks by tidal interaction. Finally, in Phase 3, almost all the HI is found outside of the galactic disks, either forming tidal tails of stripped gas (Phase 3a) or, in a few cases, in a large $\mathrm{HI}$ cloud with a single velocity gradient in which the galaxies are embedded (Phase $3 b$ ).

According to the evolutionary phases defined in Verdes-Montenegro et al. (2001), the HCGs in our sample were classified by Borthakur et al. (2010) as

- Phase 1: HCG 7, 23, 67, 79, and 88;

- Phase 2: HCG 10, 16, 25, 31, 40 1 , 58, and 100;

- Phase 3: HCG 15, 30, 37, 44, 68, 92, 93, and 97.

The evolutionary state is an indicator of the evolution of the cold ISM of the group, but it does not necessarily give the age of the group; e.g. HCG79 consists of three early-type galaxies and one intruding spiral galaxy. Stellar halo data indicates that it is an old group (Durbala et al. 2008). However, since the main part of the $\mathrm{HI}$ is located within the disk of the intruder galaxy, it is classified in evolutionary phase 1 .

\footnotetext{
1 While HCG 40 was classified in Verdes-Montenegro et al. (2001) as Phase 3, new VLA observations (Yun et al., in prep.) show that a significant amount of $\mathrm{HI}$ was missed due to a narrow spectral window, and based on these data it has been reclassified as Phase 2 .
}

We revised the velocities of the individual galaxies in the HCGs of our sample. Two galaxies not considered in Hickson et al. (1992) have been added: HCG100d, which had no velocity data in that work, and HCG31g, added to the catalog of HCGs by Rubin et al. (1990).

The basic properties of the galaxies in our sample are detailed in Table 1. The columns are

1. Galaxy: galaxy designation, following the notation of Hickson (1982).

2. $V$ : heliocentric radial velocity in $\mathrm{km} \mathrm{s}^{-1}$ (weighted average of optical measurements taken from the LEDA ${ }^{2}$ database) converted from the optical to the radio definition for comparison with the $\mathrm{CO}$ spectra.

3. $\sigma_{\mathrm{V}}$ : velocity dispersion of the galaxies in the group.

4. D: distance to the corresponding $\mathrm{HCG}$ in $\mathrm{Mpc}$, derived from the mean heliocentric velocity of the group as $D=V / H_{0}$, assuming a value of $H_{0}=75 \mathrm{~km} \mathrm{~s}^{-1} \mathrm{Mpc}^{-1}$. The mean velocity of the group is calculated averaging the velocity of the individual galaxies (Col. 2).

5. $T$ : morphological type taken from LEDA, following the RC3 classification (de Vaucouleurs et al. 1991).

6. $D_{25}$ : optical major diameter in arcmin at the $25 \mathrm{mag} \operatorname{arcsec}^{-2}$ isophot taken from LEDA.

7. $B_{\mathrm{c}}^{\mathrm{T}}$ : apparent blue magnitude taken from LEDA, corrected for Galactic dust extinction, internal extinction, and K-correction.

8. $\log \left(L_{B}\right)$ : decimal logarithm of the blue luminosity, derived from $B_{\mathrm{c}}^{\mathrm{T}}$ as

$\log \left(\frac{L_{B}}{L_{\odot}}\right)=2 \log D-0.4 B_{\mathrm{c}}^{\mathrm{T}}+11.95$.

This definition provides an estimate of the blue luminosity $\left(v L_{v}\right)$ at $4400 \AA$.

9. $\log \left(L_{K}\right)$ : decimal logarithm of the luminosity in the $K$-band in units of the solar luminosity in the $K_{\mathrm{S}}$-band $\left(L_{K, \odot}=\right.$ $\left.5.0735 \times 10^{32} \mathrm{erg} \mathrm{s}^{-1}\right)$, calculated from the extrapolated magnitude in the $K_{\mathrm{S}}(2.17 \mu \mathrm{m})$ band from the 2MASS Extended Source Catalog (Jarrett et al. 2000). We calculated the $K_{\mathrm{S}}$ luminosity, $L_{K}$, from the total (extrapolated) $K_{\mathrm{S}}$ flux, $f_{K}$, as $L_{K}=v f_{K}(v)$ (where $v$ is the frequency of the $K$-band, $\left.1.38 \times 10^{14} \mathrm{~Hz}\right)$.

10. $\log \left(M_{\mathrm{HI}}\right)$ : $\log$ arithm of the mass of the atomic hydrogen, in solar masses, for 66 of the galaxies in our sample observed with the VLA, using different combinations of the $\mathrm{C}$ and $\mathrm{D}$ configurations with beam sizes ranging from $16^{\prime \prime} \times 14^{\prime \prime}$ to $72^{\prime \prime} \times 59^{\prime \prime}$ (Verdes-Montenegro et al. 2001; and Verdes-Montenegro, priv. comm.).

${ }^{2}$ http://leda.univ-lyon1.fr/intro.html 


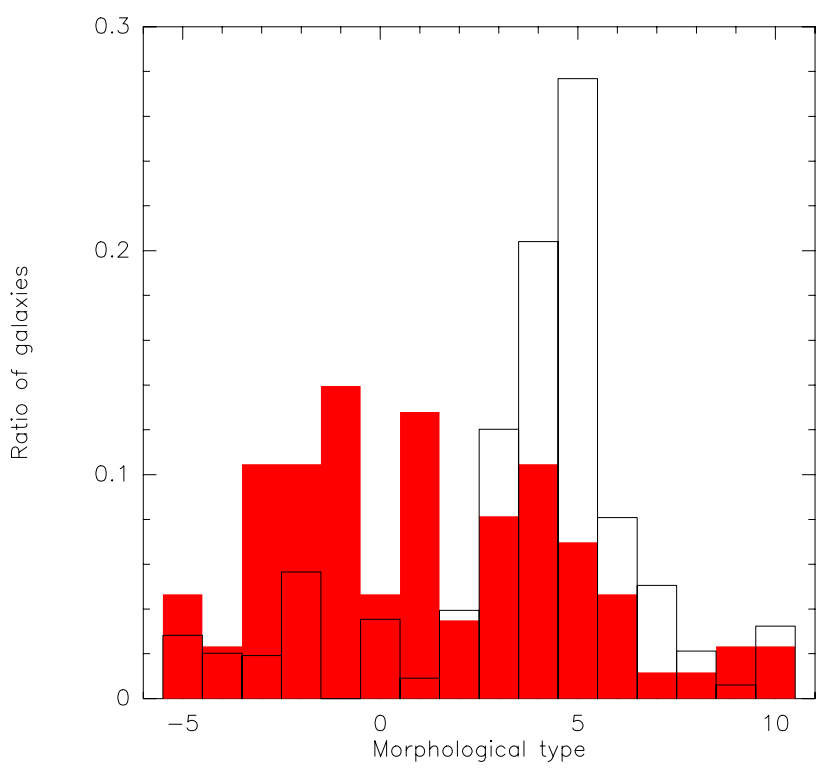

Fig. 1. Morphological types, $T(\mathrm{RC} 3)$, for the AMIGA isolated (black line) and HCGs (red filled bars) galaxies.

\subsection{Reference sample: isolated galaxies}

We chose the AMIGA sample of isolated galaxies (VerdesMontenegro et al. 2005), which is based on the CIG catalog (Karachentseva 1973), as a reference for the FIR and molecular gas properties. The FIR properties of an optically complete subsample of this catalog have been studied in Lisenfeld et al. (2007). The data that we are using for this subsample are slightly different from Lisenfeld et al. (2007) because we have taken a recent update of some basic properties into account that affects the blue magnitude, distance, morphological type, and isolation degree (detailed information provided in FernándezLorenzo 2012). We also used the $\mathrm{CO}$ data of a velocity restricted subsample $\left(1500<V<5000 \mathrm{~km} \mathrm{~s}^{-1}\right)$ of 173 AMIGA galaxies (Lisenfeld et al. 2011) to compare to our galaxies. The data for $L_{K}$ is also taken from Lisenfeld et al. (2011). When we quote mean values of the AMIGA galaxies we derived them for the subsample of galaxies with $M_{\mathrm{H}_{2}}$ data. For the analysis of the HI properties we used the work of Haynes \& Giovanelli (1984), which presents the observations and analysis of CIG galaxies, as a reference.

There are two intrinsic differences between the AMIGA and the HCG samples that must be taken into account when performing a comparison: (i) the HCG sample has a higher rate of early-type galaxies $(\sim 45 \%)$, whereas $87 \%$ of the AMIGA galaxies are spirals (Fig. 1); and (ii) the sample of AMIGA galaxies with $M_{\mathrm{H}_{2}}$ data is restricted to a velocity range of $1500<V<$ $5000 \mathrm{~km} \mathrm{~s}^{-1}$, while the range of the HCGs extends to higher velocities. Thus, the isolated galaxies are, on average, at a shorter distance (47 Mpc average distance versus $68 \mathrm{Mpc}$ for the HCG sample), which can explain their lower average luminosities (see Table 6). However, the values of the deficiencies, ratios or correlations, that we are going to discuss in the following are not expected to be affected by the difference in distance.

\section{The data}

\subsection{CO data}

We obtained CO data either by our observations or from the literature, for 86 galaxies in the selected 20 HCGs. CO data are missing for only two galaxies in these 20 groups, HCG $67 \mathrm{~d}$ and HCG 92f. The CO(1-0) line was detected for 45 galaxies.

\subsubsection{IRAM $\mathrm{CO}(1-0)$ and $\mathrm{CO}(2-1)$ observations and data reduction}

We observed 47 galaxies belonging to 14 different HCGs. The observations of the $\mathrm{CO}$ rotational transition lines $J=1 \rightarrow 0$ and $J=2 \rightarrow 1$ (at 115.271 and $230.538 \mathrm{GHz}$, respectively) were carried out with the IRAM $30 \mathrm{~m}$ radio telescope at Pico Veleta ${ }^{3}$ during June, October, and December 2006. We performed singlepointing observations using the wobbler switch mode, with a switch frequency of $0.5 \mathrm{~Hz}$ and a throw of 200". We checked for all the objects that the off-position did not coincide with a neighboring galaxy.

The dual polarization receivers A100 and B100 were used to observe the $\mathrm{CO}(1-0)$ and $\mathrm{CO}(2-1)$ lines in parallel. The median system temperature was $231 \mathrm{~K}$ for the $\mathrm{CO}(1-0)$ observations, with $\sim 80 \%$ of the galaxies observed with system temperatures between 150 and $350 \mathrm{~K}$. In the case of $\mathrm{CO}(2-1)$, the median system temperature was $400 \mathrm{~K}$, with a temperature range between 230 and $800 \mathrm{~K}$ for $85 \%$ of the galaxies. For $\mathrm{CO}(1-0)$ the $1 \mathrm{MHz}$ filterbank was used, and for $\mathrm{CO}(2-1)$ the $4 \mathrm{MHz}$ filterbank. The corresponding velocity resolutions were $2.6 \mathrm{~km} \mathrm{~s}^{-1}$ for $\mathrm{CO}(1-0)$ and $5.3 \mathrm{~km} \mathrm{~s}^{-1} \mathrm{CO}(2-1)$, respectively. The total bandwidth was $1 \mathrm{GHz}$. The half power beam width (HPBW) is $22^{\prime \prime}$ and $11^{\prime \prime}$ for 115 and $230 \mathrm{GHz}$, respectively. All CO spectra and intensities are presented on the main beam temperature scale $\left(T_{\mathrm{mb}}\right)$ which is defined as $T_{\mathrm{mb}}=\left(F_{\text {eff }} / B_{\text {eff }}\right) \times T_{\mathrm{A}}^{*}$. The IRAM forward efficiency, $F_{\text {eff }}$, was 0.95 at $155 \mathrm{GHz}$ and 0.91 at $230 \mathrm{GHz}$ and the beam efficiency, $B_{\text {eff }}$, was 0.75 and 0.54 , respectively.

The data reduction and analysis was performed using CLASS, while further analysis used GREG, both part of the GILDAS $^{4}$ package developed by IRAM. First we visually inspected the spectra and discarded bad scans. Then, spikes were removed and a constant or linear baseline was subtracted from each spectrum. The scans were then averaged to achieve a single spectrum for each galaxy and transition. These spectra were smoothed to a velocity resolution of 21 to $27 \mathrm{~km} \mathrm{~s}^{-1}$, depending on the rms. A total of 24 galaxies were detected in $\mathrm{CO}(1-0)(2$ of them marginal), 22 in $\mathrm{CO}(2-1)$ (4 of them marginal) and 18 in both transitions. The spectra are shown in Appendix A, where Fig. A.1 displays the spectra detected in $\mathrm{CO}(1-0)$ and Fig. A.2 those detected in $\mathrm{CO}(2-1)$.

For each spectrum, we integrated the intensity along the velocity interval where emission was detected. For nondetections we set an upper limit as

$I_{\mathrm{CO}}<3 \times \mathrm{rms} \times \sqrt{\delta V \Delta V}$

where $\delta V$ is the channel width and $\Delta V$ the total line width. We used a value of $\Delta V=300 \mathrm{~km} \mathrm{~s}^{-1}$ for nondetections in both $\mathrm{CO}(1-0)$ and $\mathrm{CO}(2-1)$. When the source was detected in only one transition, this line width was used to calculate the upper limit in the other transition.

The results of our $\mathrm{CO}(1-0)$ and $\mathrm{CO}(2-1)$ observations are displayed in Table 2. The columns are

1. Galaxy: galaxy designation;

2. $I_{\mathrm{CO}(1-0)}$ : velocity integrated intensity of the $\mathrm{CO}(1-0)$ emission in $\mathrm{K} \mathrm{km} \mathrm{s}^{-1}$;

${ }_{3}$ IRAM is supported by CNRS/INSU (France), the MPG (Germany) and the IGN (Spain).

${ }^{4}$ http://www.iram.fr/IRAMFR/GILDAS 
Table 2. Observed and derived molecular gas properties.

\begin{tabular}{|c|c|c|c|c|c|c|c|c|c|c|}
\hline Galaxy & $\begin{array}{c}I_{\mathrm{CO}(1-0)} \\
\left(\mathrm{K} \mathrm{km} \mathrm{s}^{-1}\right) \\
(2)\end{array}$ & $\begin{array}{l}\mathrm{rms} \\
(\mathrm{mK}) \\
(3)\end{array}$ & $\begin{array}{r}\text { Ref. }^{a} \\
\text { (4) }\end{array}$ & $\begin{array}{c}\text { HPBW } \\
(\operatorname{arcsec}) \\
(5)\end{array}$ & $\begin{array}{c}\Delta V \\
\left(\mathrm{~km} \mathrm{~s}^{-1}\right) \\
(6)\end{array}$ & $\begin{array}{c}I_{\mathrm{CO}(2-1)} \\
\left(\mathrm{K} \mathrm{km} \mathrm{s}^{-1}\right) \\
(7)\end{array}$ & $\begin{array}{c}\mathrm{rms} \\
(\mathrm{mK}) \\
(8)\end{array}$ & $\begin{array}{c}\Delta V \\
\left(\mathrm{~km} \mathrm{~s}^{-1}\right) \\
(9)\end{array}$ & $\begin{array}{c}\log \left(M_{\mathrm{H}_{2} \text { obs }}\right) \\
\left(M_{\odot}\right) \\
(10)\end{array}$ & $\begin{array}{c}\log \left(M_{\mathrm{H}_{2}}\right) \\
\left(M_{\odot}\right) \\
(11)\end{array}$ \\
\hline $7 a$ & 7.20 & & 3 & 43 & 500 & & & & 9.51 & 9.71 \\
\hline $7 b$ & $<0.70$ & & 3 & 55 & $\ldots$ & & & & $<8.71$ & $<8.80$ \\
\hline $7 \mathrm{c}$ & 1.40 & & 3 & 55 & 183 & & & & 9.01 & 9.17 \\
\hline $7 \mathrm{~d}$ & $<0.60$ & & 3 & 43 & $\ldots$ & & & & $<8.43$ & $<8.52$ \\
\hline $10 \mathrm{a}$ & $2.72 \pm 0.49$ & & 2 & 22 & 339 & & & & 8.79 & 9.51 \\
\hline$\ldots$ & $\ldots$ & $\ldots$ & $\ldots$ & $\ldots$ & $\ldots$ & $\ldots$ & $\ldots$ & $\ldots$ & $\ldots$ & $\ldots$ \\
\hline
\end{tabular}

Notes. The full table is available in electronic form at the CDS and from http://amiga.iaa.es. ${ }^{(a)}$ CO reference code: 1: Our observations. 2: Leon et al. (1998). 3: Verdes-Montenegro et al. (1998).

3. rms: root-mean-square noise of the $\mathrm{CO}(1-0)$ spectrum (if available) in $\mathrm{mK}$;

4. Ref.: reference of the $\mathrm{CO}(1-0)$ data, detailing whether data come from our observations or from the literature (see Sect. 3.1.2);

5. Beam: HPBW of the telescope in arcsecond;

6. $\Delta V_{\mathrm{CO}(1-0)}$ : line width of the $\mathrm{CO}(1-0)$ emission (if detected) in $\mathrm{km} \mathrm{s}^{-1}$;

7. $I_{\mathrm{CO}(2-1)}$ : velocity integrated intensity of the $\mathrm{CO}(2-1)$ emission (if observed) in $\mathrm{K} \mathrm{km} \mathrm{s}^{-1}$;

8. rms: rms of the $\mathrm{CO}(2-1)$ spectrum (if observed) in $\mathrm{mK}$;

9. $\Delta V_{\mathrm{CO}(2-1)}$ : line width of the $\mathrm{CO}(2-1)$ emission (if detected) in $\mathrm{km} \mathrm{s}^{-1}$;

10. $\log \left(M_{\mathrm{H}_{2} \text { obs }}\right)$ : $\log$ arithm of the $\mathrm{H}_{2}$ mass (in solar masses) calculated from the observed central $I_{\mathrm{CO}}$ (see Sect. 3.1.3);

11. $\log \left(M_{\mathrm{H}_{2}}\right)$ : $\log$ arithm of the $\mathrm{H}_{2}$ mass (in solar masses) extrapolated to the emission from the total disk (see Sect. 3.1.3).

\subsection{2. $\mathrm{CO}(1-0)$ data from the literature}

We have searched in the literature for available $\mathrm{CO}(1-0)$ data for the 20 HCGs of our sample and have compiled data for the velocity-integrated $\mathrm{CO}(1-0)$ intensities and line widths (also listed in Table 2) from the following sources:

- Verdes-Montenegro et al. (1998): 24 galaxies from nine different HCGs. Twenty of them were observed with the NRAO $12 \mathrm{~m}$ telescope at Kitt Peak with a beam size of 55". The data from the four other galaxies are from Boselli et al. (1996), observed with the SEST $15 \mathrm{~m}$ telescope, with a 43" beam. Two of these galaxies (68d and 88c) were also observed by us, but we chose the Verdes-Montenegro et al. (1998) data because of their better quality;

- Leon et al. (1998): 17 galaxies corresponding to ten different HCGs, observed with the IRAM $30 \mathrm{~m}$ telescope with a similar setting as in our observations (see Sect. 3.1.1).

There are 16 galaxies that were observed both by us and by Verdes-Montenegro et al. (1998) or Leon et al. (1998). Furthermore, 14 were observed by both Verdes-Montenegro et al. (1998) and Leon et al. (1998). To choose between the different existing spectra (either from our observations or from the literature), we first checked that they were consistent and then applied the following criteria: if available, we chose the spectrum with detected emission. If more than one detected spectrum existed, we chose the one with the lower rms or - in case of comparable rms- the spectrum observed with a larger beam, in order to probe a larger fraction of the disk. Except for the two galaxies mentioned above, we always selected our data due to their better quality in case of duplication. In total, we have $\mathrm{CO}(1-0)$ spectra for 86 galaxies ( 45 from our own observations and 41 from the literature) for our statistical analysis.

\subsubsection{Molecular gas mass}

We calculate the molecular gas mass, $M_{\mathrm{H}_{2}}$ using the following equation:

$M_{\mathrm{H}_{2}}=75 \times D^{2} I_{\mathrm{CO}(1-0)} \Omega$

where $\Omega$ is the area covered by the observations in $\operatorname{arcsec}^{2}$ (i.e. $\Omega=1.13 \theta^{2}$ for a single pointing with a Gaussian beam where $\theta$ is the HPBW). This equation assumes a CO-to- $\mathrm{H}_{2}$ conversion factor $X=N_{\mathrm{H}_{2}} / I_{\mathrm{CO}}=2 \times 10^{20} \mathrm{~cm}^{-2}\left(\mathrm{~K} \mathrm{~km} \mathrm{~s}^{-1}\right)^{-1}$ (e.g. Dickman et al. 1986). No correction factor for the fraction of helium and other heavy metals is included. The molecular gas masses of the AMIGA galaxies are calculated in the same way.

In both the observations that we carried out and the data from the literature, a single position at the center of the galaxy was observed. Because of this and the different beams used by us and others we need to correct for possible emission outside the beam. To extrapolate the observed CO intensities to the total value within the disk we need to know the distribution and extension of the CO emission. Different authors (Nishiyama et al. 2001; Regan et al. 2001; Leroy et al. 2008) have found that the velocity-integrated $\mathrm{CO}$ intensity in spiral galaxies follows an exponential distribution as a function of radius with a scale length $r_{\mathrm{e}}$ :

$I_{\mathrm{CO}}(r)=I_{0} \propto \exp \left(r / r_{\mathrm{e}}\right)$.

We adopt a scale length of $r_{\mathrm{e}}=0.2 \times r_{25}$, where $r_{25}$ is the major optical 25 mag $\operatorname{arcsec}^{-2}$ isophotal radius, following Lisenfeld et al. (2011), who derive this scale length from studies of the mentioned authors and from their own CO data. We used this distribution to calculate the expected $\mathrm{CO}$ emission from the entire disk, taking the galaxy inclination into account (see Lisenfeld et al. 2011, for more details). This approach assumes that the distribution of the molecular gas in galaxies in HCGs is the same as in field spiral galaxies. The implications of this approach are discussed below.

The resulting aperture correction factor for $M_{\mathrm{H}_{2}}$ (defined as the ratio between $M_{\mathrm{H}_{2}}$ observed in the central pointing and $M_{\mathrm{H}_{2}}$ extrapolated to the entire disk) is shown in Fig. 2. The ratio between the extrapolated and central intensities is below two for most galaxies (66 out of 86 , or $77 \%$ ), with an average value of 1.78 . To check the consistency of the extrapolation, we also performed the analysis presented in this paper for a sample restricted to galaxies with a small (less than a factor 1.6) aperture correction $(n=45)$, finding no significant differences with 


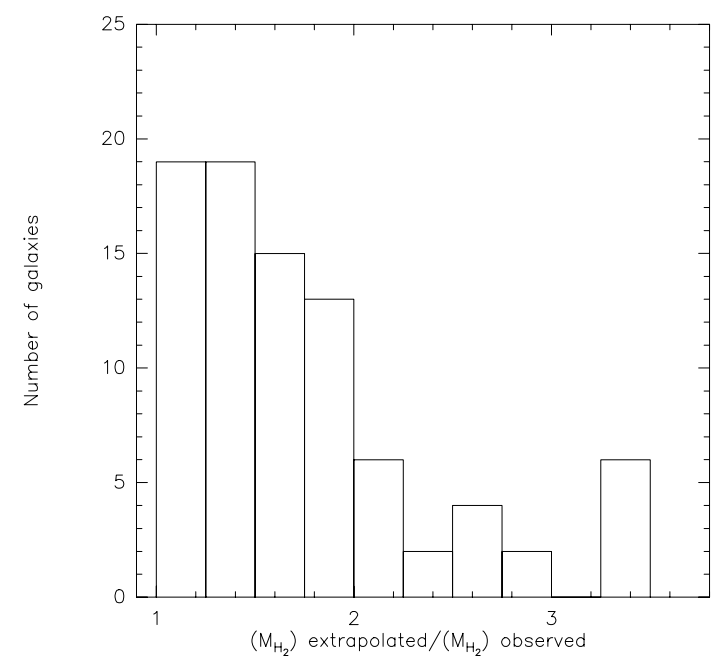

Fig. 2. Distribution of the aperture correction factor for $M_{\mathrm{H}_{2}}$.

respect to the full sample. Thus, we conclude that the aperture correction does not introduce any bias into the results.

The values for the molecular gas mass in the central pointing and the extrapolated molecular gas mass are listed in Table 2. Here, and in the following, we always use the extrapolated molecular gas mass and denote it as $M_{\mathrm{H}_{2}}$ for simplicity. The $M_{\mathrm{H}_{2}}$ distribution is shown in Fig. 3. The average value for spiral galaxies $(T \geq 1)$ is listed in Table 6 . The distribution and average values of $M_{\mathrm{H}_{2}}$, as well as the statistical distributions and average values throughout this work, have been calculated using the Kaplan-Meier estimator implemented in $\mathrm{ASURV}^{5}$, to take the upper limits in the data into account.

\subsection{Far-infrared data}

FIR fluxes were obtained from ADDSCAN/SCANPI, a utility provided by the Infrared Processing and Analysis Center (IPAC) ${ }^{6}$. This is a one-dimensional tool that coadds calibrated survey data of the Infrared Astronomical Satellite (IRAS). It makes use of all scans that passed over a specific position and produces a scan profile along the average scan direction. It is three to five times more sensitive than the IRAS Point Source Catalog (PSC) since it combines all survey data, so it is more suitable for detection of the total flux from slightly extended objects.

We have compiled the FIR data (also derived using ADDSCAN/SCANPI) for 63 galaxies in our sample from Verdes-Montenegro et al. (1998). In the case of the remaining 23 galaxies, we derived FIR fluxes directly using ADDSCAN/SCANPI. To choose the best flux estimator we have followed the guidelines given in the IPAC website ${ }^{7}$, which are explained in Lisenfeld et al. (2007). As a consistency check, we also applied this procedure to 14 galaxies in the list of VerdesMontenegro et al. (1998). We found no significant differences, with an average difference of $15 \%$ between our reprocessed fluxes and those in Verdes-Montenegro et al. (1998).

\footnotetext{
5 Astronomical Survival Analysis (ASURV) Rev. 1.1 (Lavalley et al. 1992) is a generalized statistical package that implements the methods presented by Feigelson \& Nelson (1985).

${ }^{6}$ http://scanpi.ipac.caltech.edu: $9000 /$

7 http://irsa.ipac.caltech.edu/IRASdocs/scanpi_interp. html
}
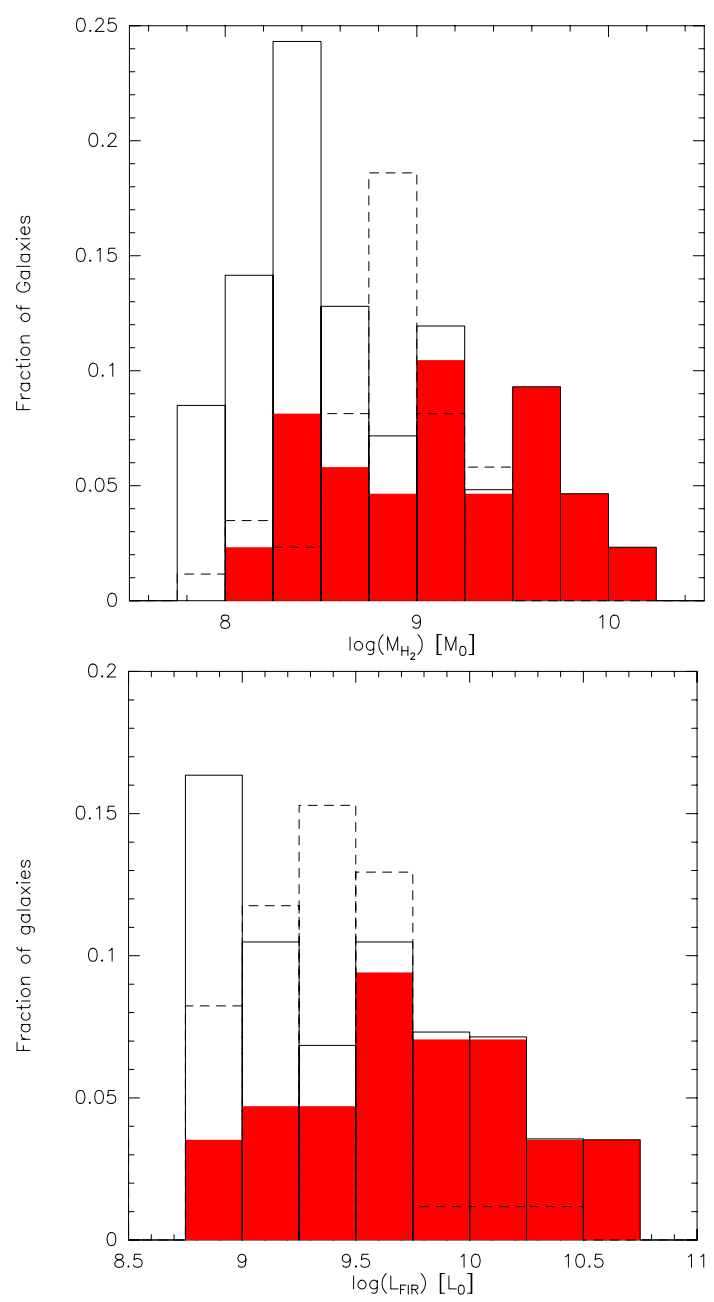

Fig. 3. $M_{\mathrm{H}_{2}}$ and $L_{\mathrm{FIR}}$ distribution of the HCG galaxies. The red filled bins show the distribution of the detected galaxies, the dashed line gives the distribution of upper limits and the full line shows the distribution calculated with ASURV.

From the fluxes at 60 and $100 \mu \mathrm{m}$ the FIR luminosity, $L_{\mathrm{FIR}}$, is computed as

$\log \left(L_{\mathrm{FIR}} / L_{\odot}\right)=\log (\mathrm{FIR})+2 \log (D)+19.495$,

where FIR is defined as (Helou et al. 1988):

FIR $=1.26 \times 10^{-14}\left(2.58 F_{60}+F_{100}\right) \mathrm{W} \mathrm{m}^{-2}$.

The computed $L_{\mathrm{FIR}}$, together with the 60 and $100 \mu$ m fluxes compiled from ADDSCAN/SCANPI, are detailed in Table 3. The distribution of $L_{\mathrm{FIR}}$ is shown in Fig. 3. The average value of $L_{\mathrm{FIR}}$ for spiral galaxies is given in Table 6 .

For galaxies HCG 31a and HCG 31c, the FIR fluxes could not be separated. Therefore, we use the sum of both. When comparing $L_{\mathrm{FIR}}$ to other magnitudes $\left(L_{B}, M_{\mathrm{H}_{2}}\right.$, or $\left.M_{\mathrm{HI}}\right)$, we also use the sum of both galaxies.

In order to check the accuracy of the low resolution IRAS data, we compared them to $24 \mu \mathrm{m}$ data from Spitzer for the 12 groups for which Spitzer data is available. We compared the SFR derived from $L_{\mathrm{FIR}}$ (calculated from Eq. (10)) to that derived from the Spitzer $24 \mu \mathrm{m}$ luminosity, $L_{24} \mu \mathrm{m}$, (from Bitsakis et al. 2010, 2011) using the equation $\operatorname{SFR}\left(M_{\odot} \mathrm{yr}^{-1}\right)=8.10 \times$ $10^{-37}\left(L_{24 \mu \mathrm{m}}\left(\mathrm{erg} \mathrm{s}^{-1}\right)\right)^{0.848}$ (from Calzetti et al. 2010). For most of the objects the agreement was satisfactory: the values of the SFR derived in both ways agreed to better than a factor 2.5 
Table 3. FIR, SFR, SFE, and SSFR.

\begin{tabular}{lccccccc}
\hline \hline Galaxy & Ref. $^{a}$ & $\begin{array}{c}I_{60} \\
(\mathrm{Jy})\end{array}$ & $\begin{array}{c}I_{100} \\
(\mathrm{Jy})\end{array}$ & $\begin{array}{c}\log \left(L_{\mathrm{FIR}}\right) \\
\left(L_{\odot}\right)\end{array}$ & $\begin{array}{c}S F R \\
\left(M_{\odot} \mathrm{yr}^{-1}\right)\end{array}$ & $\begin{array}{c}\log (\mathrm{SFE})^{b} \\
\left(\mathrm{yr}^{-1}\right)\end{array}$ & $\begin{array}{c}\log (\mathrm{sSFR}) \\
\left(M_{\odot} \mathrm{yr}^{-1}\right)\end{array}$ \\
\hline $7 \mathrm{a}$ & 2 & 3.32 & 6.61 & 10.23 & 3.75 & -9.14 & -10.33 \\
$7 \mathrm{~b}$ & 2 & $<0.18$ & $<0.32$ & $<8.95$ & $<0.20$ & & $<-11.30$ \\
$7 \mathrm{c}$ & 2 & 0.61 & 2.35 & 9.65 & 0.99 & -9.18 & -10.62 \\
$7 \mathrm{~d}$ & 2 & $<0.15$ & $<0.39$ & $<8.95$ & $<0.20$ & & $<-10.63$ \\
$10 \mathrm{a}$ & 2 & 0.50 & 1.81 & 9.72 & 1.16 & -9.45 & -11.02 \\
$\ldots$ & $\ldots$. & $\ldots$ & $\ldots$. & $\ldots$. & $\ldots$. & $\ldots$. & $\ldots$. \\
\hline
\end{tabular}

Notes. The full table is available in electronic form at the CDS and from http://amiga.iaa.es. ${ }^{(a)}$ Reference code (see Sect. 3.2): 1: our data analysis. 2: Verdes-Montenegro et al. (1998). ${ }^{(b)}$ The value of the SFE is not displayed for the galaxies with upper limits in both $L_{\mathrm{FIR}}$ and $M_{\mathrm{H}_{2}}$.

or, in case of IRAS upper limits, the resulting upper limits for the SFR were above those derived from $L_{24} \mu \mathrm{m}$. There were only three galaxies in two groups with a larger discrepancy. For HCG $79 \mathrm{~b}$ and for HCG $37 \mathrm{~b}$ we obtained a value of the SFR derived from IRAS that was a factor of six higher than the SFR from the $24 \mu \mathrm{m}$ data and for HCG $37 \mathrm{a}$ the difference was a factor of ten. After checking the Spitzer images and IRAS data, we found that in the case of HCG 79 the reason for the discrepancy was the blending of HCG 79a and 79b in the IRAS beam. We thus assumed that the value of $L_{\mathrm{FIR}}$ given for HCG 79b in Verdes-Montenegro et al. (1998) arises from both galaxies, so we assigned to each object a fraction of the IRAS fluxes and $L_{\text {FIR }}$ such that $\operatorname{SFR}(\operatorname{IRAS})=\operatorname{SFR}(24 \mu \mathrm{m})$. A similar situation occurred in the case of HCG 37, where three objects (HCG 37a, HCG 37b and HCG 37c) are blended in the IRAS beam. Here, we assumed that the value of $L_{\mathrm{FIR}}$ given for HCG 37b in Verdes-Montenegro et al. (1998) was emitted from all three galaxies and corrected in the same way as for HCG 79.

\section{Results}

In this section, we aim to study the relation between $M_{\mathrm{H}_{2}}$ and the SFR in HCG galaxies and compare them to isolated galaxies. Furthermore, we search for relations with the atomic gas deficiency of the galaxies and the groups and with the evolutionary phase of the groups. We furthermore investigate the ratio between the two $\mathrm{CO}$ transitions, $\mathrm{CO}(1-0)$ and $\mathrm{CO}(2-1)$.

To search for differences to isolated galaxies, we used two methods: (i) we normalized $M_{\mathrm{H}_{2}}$ and $L_{\mathrm{FIR}}$ to the blue luminosity, $L_{B}$, or the luminosity in the $K$-band, $L_{K}$, and compared the ratios to those of isolated galaxies, and (ii) we calculated the deficiency parameters of $M_{\mathrm{H}_{2}}, L_{\mathrm{FIR}}$ and $M_{\mathrm{HI}}$ of the galaxies (see Sect. 4.2). In general, we obtained very consistent results for $L_{B}$ and $L_{K}$.

We carry out this analysis separately for early-type galaxies and spirals for the following reasons. (i) The morphological distribution is very different for both samples with a much higher fraction of early-type galaxies among HCG galaxies. (ii) The number of early-type galaxies in the AMIGA reference sample is very small so that no statistically significant comparison sample is available. In particular, no deficiency parameter can be derived. (iii) Early-type galaxies tend to have a significantly lower molecular gas content than late-type galaxies, and their FIR emission is not as clearly related to their SFR as it is in latetype galaxies, as a result of the lack of strong SF. Therefore, the use of $L_{\mathrm{FIR}}$ as an SF tracer is more questionable.

\subsection{Relation between $M_{\mathrm{H}_{2}}, L_{\mathrm{FIR}}, M_{\mathrm{HI}}$, and $L_{\mathrm{B}}$}

Figure 4 shows $M_{\mathrm{H}_{2}}$ (top) and $L_{\mathrm{FIR}}$ (bottom) versus $L_{B}$ for spirals galaxies (left) and early-type galaxies (right). For spiral galaxies

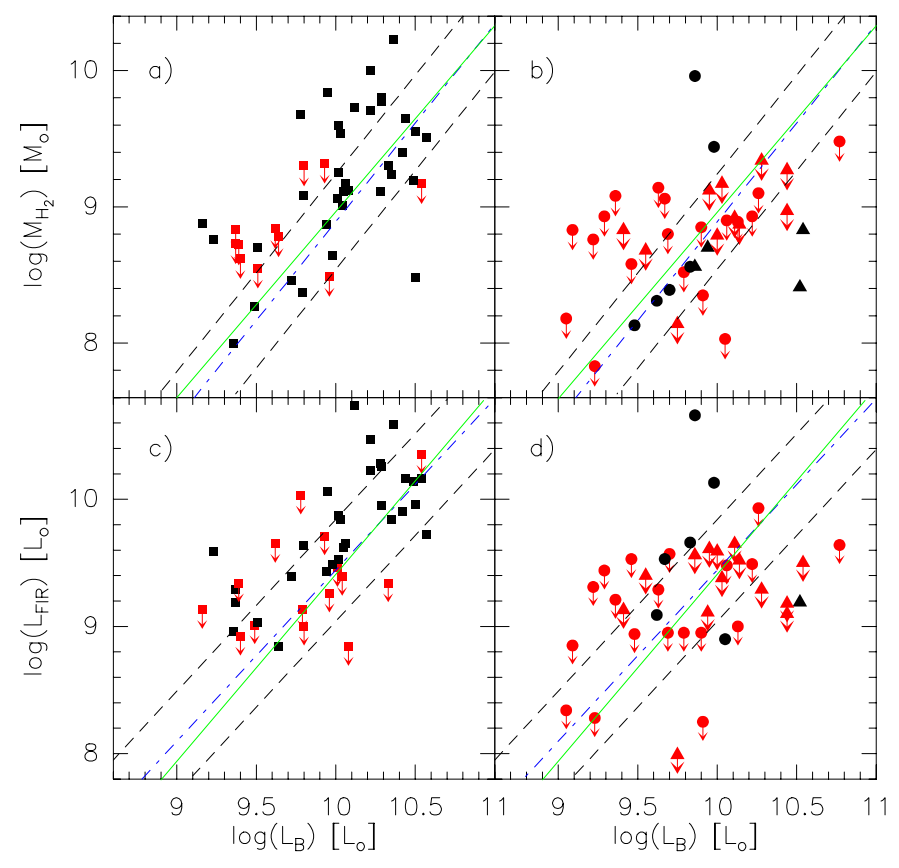

Fig. 4. $M_{\mathrm{H}_{2}}$ vs. $L_{B}$ for a) spiral galaxies $(T \geq 1)$ and b) elliptical (circles) and $\mathrm{S} 0$ galaxies (triangles). $L_{\mathrm{FIR}}$ vs. $L_{B}$ for c) spiral galaxies $(T \geq 1)$ and d) elliptical (circles) and S0 galaxies (triangles). The full green line corresponds to the bisector fit found for HCG galaxies (fit parameters are given in Table 4), while the blue dashed-dotted line corresponds to the bisector fit found for the AMIGA isolated galaxies. Both fits are done for the entire range of morphological types. The dashed black lines are offset by the standard deviation of the correlation for the isolated galaxies, which is \pm 0.35 for the $M_{\mathrm{H}_{2}}$ and \pm 0.4 for $L_{\mathrm{FIR}}$. Black symbols denote detections and red symbols upper limits.

good correlations exist between both $M_{\mathrm{H}_{2}}, L_{\mathrm{FIR}}$ and $L_{B}$. A linear fit to the total sample of HGCs is plotted, along with the corresponding fit to the AMIGA sample. The coefficients are listed in Table 4 . The fits on $L_{\mathrm{FIR}}$ vs. $L_{B}$ for the AMIGA sample are slightly different from the values in Lisenfeld et al. (2007) because we have considered a recent update of the basic properties of the galaxies (e.g. distance and morphological type; see Fernández-Lorenzo 2012, for more details). A slight shift towards higher values in $M_{\mathrm{H}_{2}}$ seems to be present in comparison to the best-fit line of isolated galaxies. The linear regressions between $L_{\mathrm{FIR}}$ and $L_{B}, M_{\mathrm{H}_{2}}$ and $L_{B}$, or $M_{\mathrm{H}_{2}}$ and $L_{\mathrm{FIR}}$ (Table 4) show no significative differences between HCGs and isolated galaxies. No clear correlation is visible for early-type galaxies and for $\log \left(L_{B}\right) \gtrsim 10$, the values of both $M_{\mathrm{H}_{2}}$ and $L_{\mathrm{FIR}}$ are below those of spiral galaxies.

We note that, in contrast to $M_{\mathrm{H}_{2}}$ and $L_{\mathrm{FIR}}, M_{\mathrm{HI}}$ shows no correlation with $L_{B}$ (Fig. 5), reflecting that $\mathrm{HI}$ is very strongly 
Table 4. Correlation analysis of $M_{\mathrm{H}_{2}}$ vs. $L_{B}, L_{\mathrm{FIR}}$ vs. $L_{B}$, and $M_{\mathrm{H}_{2}}$ vs. $L_{\mathrm{FIR}}$.

\begin{tabular}{lcccccc}
\hline \hline Magnitude & Sample & & $\begin{array}{c}\text { Slope } \\
\text { (bisector) }\end{array}$ & $\begin{array}{c}\text { Intercept } \\
\text { (bisector) }\end{array}$ & $\begin{array}{c}\text { Slope } \\
\left(L_{B} \text { indep. }\right)\end{array}$ & $\begin{array}{c}\text { Intercept } \\
\left(L_{B} \text { indep. }\right)\end{array}$ \\
\hline$M_{\mathrm{H}_{2}}$ vs. $L_{B}$ & HCGs & All & $1.37 \pm 0.15$ & $-4.74 \pm 1.48$ & $0.81 \pm 0.14$ & $0.73 \pm 1.35$ \\
& & $T>0$ & $1.40 \pm 0.16$ & $-4.94 \pm 1.61$ & $0.95 \pm 0.20$ & $-0.43 \pm 1.97$ \\
& AMIGA & & $1.45 \pm 0.08$ & $-5.61 \pm 0.77$ & $1.12 \pm 0.08$ & $-2.43 \pm 0.83$ \\
\hline$L_{\mathrm{FIR}}$ vs. $L_{B}$ & HCGs & All & $1.47 \pm 0.16$ & $-5.29 \pm 1.54$ & $0.79 \pm 0.15$ & $1.43 \pm 1.49$ \\
& & $T>0$ & $1.31 \pm 0.16$ & $-3.37 \pm 1.99$ & $0.77 \pm 0.16$ & $2.00 \pm 1.58$ \\
& AMIGA & & $1.35 \pm 0.04$ & $-4.06 \pm 0.37$ & $1.12 \pm 0.04$ & $-1.73 \pm 0.38$ \\
\hline$M_{\mathrm{H}_{2}}$ vs. $L_{\mathrm{FIR}}$ & HCGs & All & $0.90 \pm 0.09$ & $0.41 \pm 0.83$ & $0.75 \pm 0.09$ & $1.82 \pm 0.86$ \\
& & $T>0$ & $1.21 \pm 0.11$ & $-2.63 \pm 1.11$ & $1.04 \pm 0.11$ & $-1.00 \pm 1.08$ \\
& AMIGA & & $1.16 \pm 0.08$ & $-2.14 \pm 0.72$ & $0.98 \pm 0.06$ & $-0.46 \pm 0.61$ \\
\hline
\end{tabular}

Notes. The slope and intercept are defined as $\log \left(M_{\mathrm{H}_{2}}\right)=\log \left(L_{B}\right) \times$ slope + intercept, $\log \left(L_{\mathrm{FIR}}\right)=\log \left(L_{B}\right) \times$ slope + intercept and $\log \left(M_{\mathrm{H}_{2}}\right)=$ $\log \left(L_{\mathrm{FIR}}\right) \times$ slope + intercept. The AMIGA fits involving $M_{\mathrm{H}_{2}}$ are taken from Lisenfeld et al. (2011).

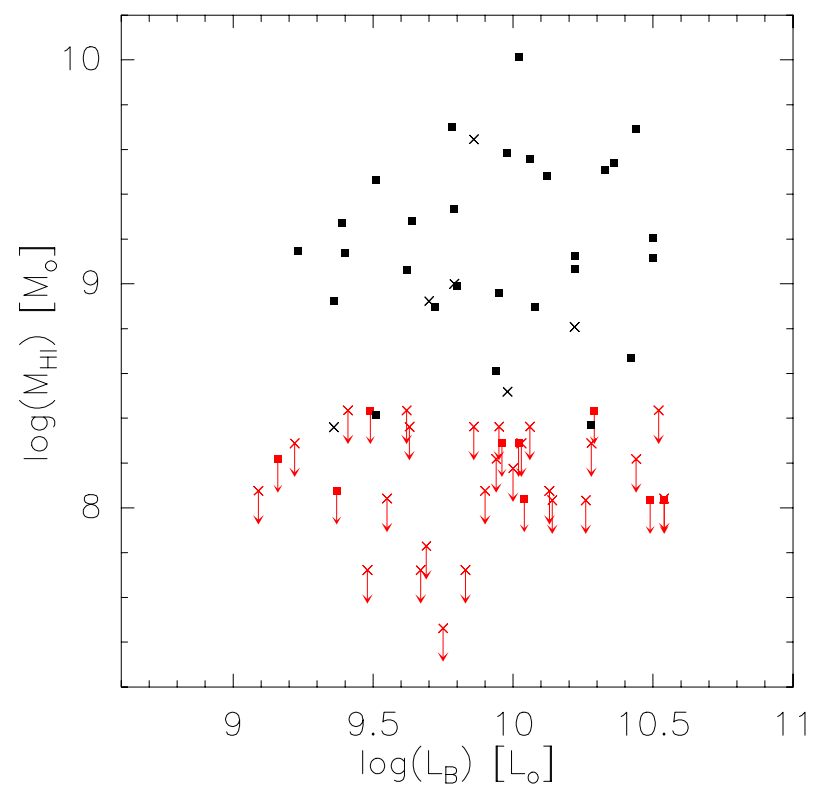

Fig. 5. $M_{\mathrm{HI}}$ vs. $L_{B}$ for late-type ( $T \geq 1$, squares) and early-type (crosses) galaxies. Black symbols denote detections and red symbols upper limits.

affected by the interactions and in many galaxies of our evolved groups largely removed from the galaxies.

Previous surveys (see e.g. Young \& Scoville 1991) have found a linear correlation between $M_{\mathrm{H}_{2}}$ and $L_{\mathrm{FIR}}$. A linear correlation can also be seen in our sample (Fig. 6). We include in this figure the lines for constant $L_{\mathrm{FIR}} / M_{\mathrm{H}_{2}}$ values equal to 1,10 , and $100 L_{\odot} / M_{\odot}$. Practically all of our galaxies lie in the range of $L_{\mathrm{FIR}} / M_{\mathrm{H}_{2}}=1-10 L_{\odot} / M_{\odot}$, which is typical of normal, quiescent galaxies (Young \& Scoville 1991).

Finally, we have directly compared E and S0 galaxies in HCGs to galaxies of the same types in the AMIGA sample. In the case of lenticular galaxies we limited the sample of galaxies in HCGs to the same distance range as the AMIGA sample (40$70 \mathrm{Mpc}$ ) since for the largest distances the rate of upper limits is very high in HCGs and does not provide any further information. In Fig. 7 (top) we show the relation between $M_{\mathrm{H}_{2}}$ and $L_{B}$ for the SOs in HCGs and from the AMIGA sample. Even though the number of data points is low, a trend seems to be present for S0s in isolated galaxies to have a higher $M_{\mathrm{H}_{2}}$ for the same $L_{B}$. A similar result is found for $L_{\mathrm{FIR}}$ (not shown here), where most lenticular isolated galaxies present higher values than expected for their

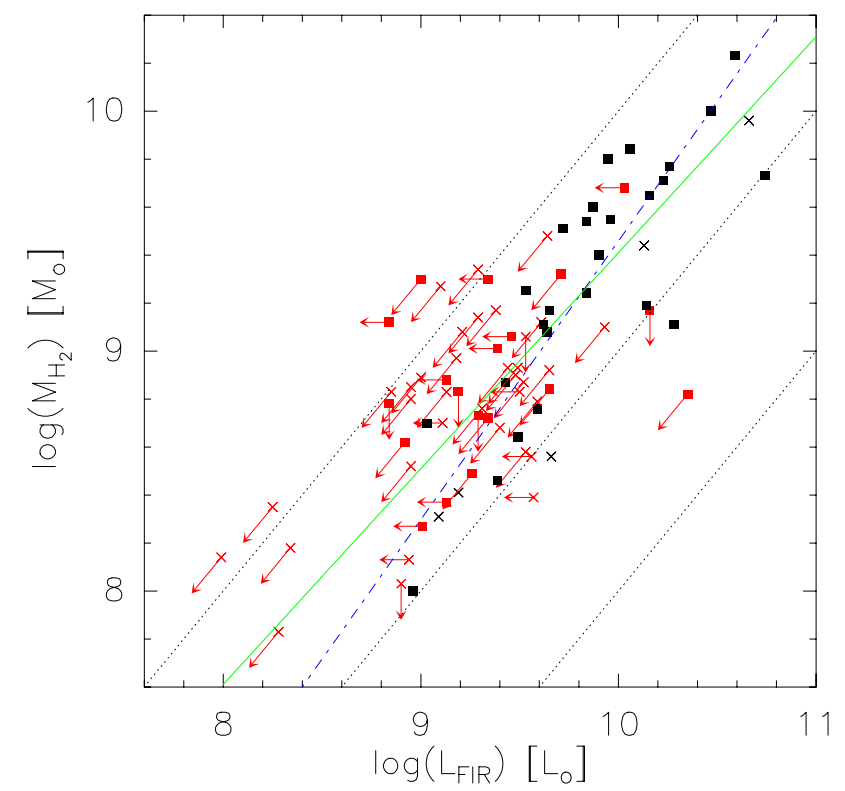

Fig. 6. $M_{\mathrm{H}_{2}}$ vs. $L_{\mathrm{FIR}}$ for late-type ( $T \geq 1$, squares) and early-type (E+S0, crosses) galaxies. The green line corresponds to the bisector fit found for HCGs galaxies, while the blue dashed-dotted line corresponds to the bisector fit found for the AMIGA isolated galaxies from Lisenfeld et al. (2011). The fits are detailed in Table 4. The dotted black lines correspond to the $L_{\mathrm{FIR}} / M_{\mathrm{H}_{2}}$ ratios 1 (left), 10 (middle), and 100 (right) $L_{\odot} / M_{\odot}$. Black symbols denote detections and red symbols upper limits.

optical luminosity, while most of the objects in HCGs show upper limits excluding any excess. If SO galaxies in these dense environments originate from stripping of spirals, this might indicate that molecular gas has also been removed in the process. Although this interpretation is speculative owing to the lowquality statistics, it provides hints for further research in future works.

Concerning the elliptical galaxies, none of the isolated galaxies is detected in CO, while among the four detections in HCGs two have a mass similar to the expected for spiral isolated galaxies (HCG 15d and HCG 79b), and the other two show significantly lower masses (HCG 37a and HCG 93a), pointing to an external origin (Fig. 7, bottom). The FIR luminosity of the Es in HCGs (not shown here) is similar to what is expected for spiral galaxies. It is also noticeable that, while the range of $L_{B}$ values for the S0s in HCGs covers about the same range as for isolated galaxies, Es in HCGs are up to half an order of magnitude more luminous than isolated Es. 
V. Martinez-Badenes et al.: Molecular gas content and SFR in Hickson compact groups

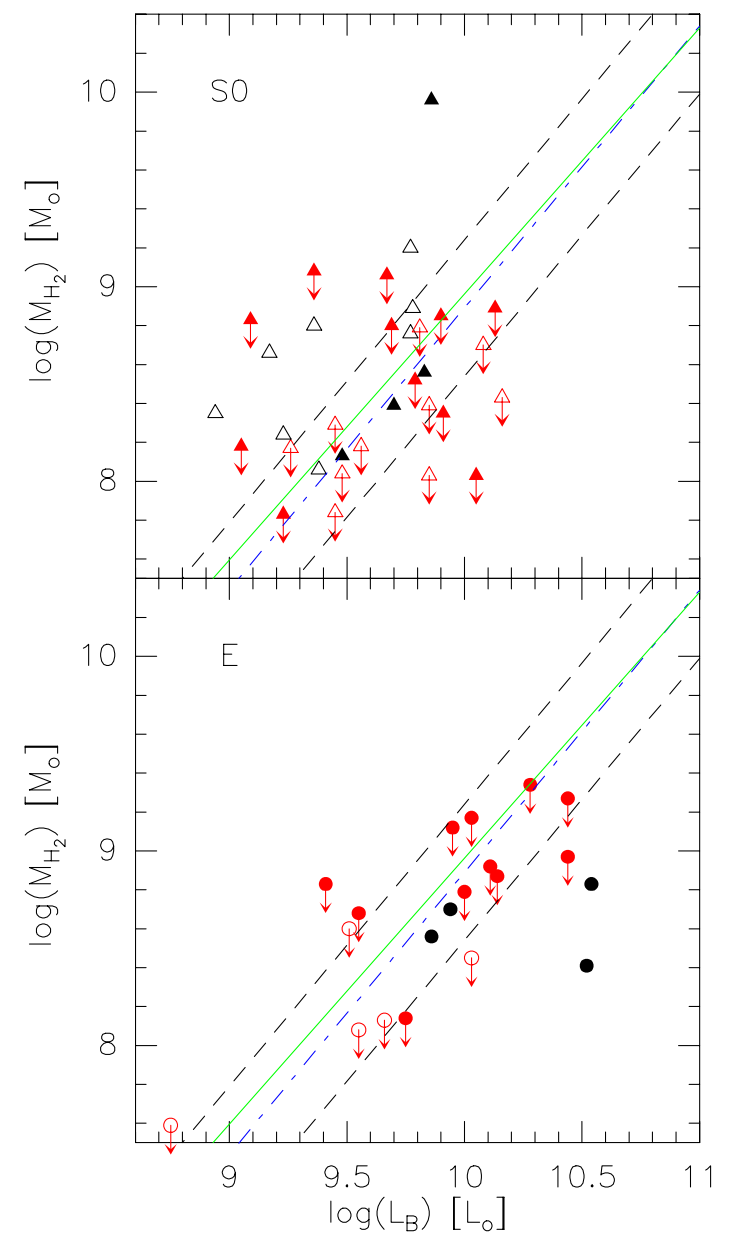

Fig. 7. $M_{\mathrm{H}_{2}}$ vs. $L_{B}$ for early-type galaxies in HCGs (full symbols) and from the AMIGA sample of isolated galaxies (open symbols) with distances between 20 and $70 \mathrm{Mpc}$. The lines are the same as in Fig. 4. Black symbols denote detections and red symbols upper limits. Top: S0 galaxies (triangles); bottom: elliptical galaxies (circles).

\subsection{Deficiencies}

We have calculated the $M_{\mathrm{H}_{2}}, L_{\mathrm{FIR}}$, and $M_{\mathrm{HI}}$ deficiencies following the definition of Haynes \& Giovanelli (1984) as

$\operatorname{Def}(X)=\log \left(X_{\text {predicted }}\right)-\log \left(X_{\text {observed }}\right)$

where we calculated the predicted value of the variable $X$ from $L_{B}$. Following this definition, a negative deficiency implies an excess with respect to the predicted value.

The expected $M_{\mathrm{H}_{2}}$ for each galaxy is calculated from its $L_{B}$ using the fit to the AMIGA sample in Lisenfeld et al. (2011). The fit, which is given in Table 4, was calculated without distinguishing morphological types. The dominance of spiral galaxies in the AMIGA sample means that the fit is only adequate for spiral galaxies. Because of the low number of early-type galaxies in the AMIGA sample, it is not possible to derive a meaningful deficiency parameter for them. In addition, we calculated the deficiency derived from the relation between $M_{\mathrm{H}_{2}}$ and $L_{K}$ of the AMIGA sample (Lisenfeld et al. 2011), $\log \left(M_{\mathrm{H}_{2}}\right)=-2.27+$ $1.05 \times \log \left(L_{K}\right)$. In a similar way, the expected $L_{\mathrm{FIR}}$ is calculated from the fit between $L_{\mathrm{FIR}}$ and $L_{B}$ obtained for the AMIGA isolated galaxies (Table 4 ) for the sample presented in Lisenfeld et al. (2007).

The correlations between $M_{\mathrm{H}_{2}}\left(L_{\mathrm{FIR}}\right)$ and $L_{B}$, or $L_{K}$, have a considerable scatter with standard deviations of 0.35 dex for
Table 5. Deficiencies of $M_{\mathrm{H}_{2}}, L_{\mathrm{FIR}}$, and $M_{\mathrm{HI}}$ derived from $L_{B}$.

\begin{tabular}{lccc}
\hline \hline Galaxy & $\operatorname{Def}\left(M_{\mathrm{H}_{2}}\right)$ & $\operatorname{Def}\left(L_{\mathrm{FIR}}\right)$ & $\operatorname{Def}\left(M_{\mathrm{HI}}\right)$ \\
\hline $7 \mathrm{a}$ & -0.50 & -0.49 & 0.67 \\
$7 \mathrm{~b}$ & $>-0.36$ & $>0.07$ & $>1.38$ \\
$7 \mathrm{c}$ & -0.19 & -0.13 & 0.29 \\
$7 \mathrm{~d}$ & $>-0.06$ & $>0.21$ & 0.28 \\
$10 \mathrm{a}$ & 0.21 & 0.49 & - \\
$\ldots$. & $\ldots$. & $\ldots$. & $\ldots$. \\
\hline
\end{tabular}

Notes. The full table is available in electronic form at the CDS and from http://amiga.iaa.es.

$M_{\mathrm{H}_{2}}$ and 0.4 dex for $L_{\mathrm{FIR}}$. These standard deviations are much higher than the observational measurement errors. In this case, the error of the mean values are completely dominated by the statistical errors, so we neglect the observational errors in our calculations. The high standard deviation means that individual galaxies with deficiencies up to about these values represent normal deviations from the mean. However, the much smaller error of the mean deficiency allows samples of galaxies (here: galaxies in HCGs and isolated galaxies) to be compared with a much higher precision.

The HI deficiency of the galaxies is calculated following the morphology-dependent fit between $M_{\mathrm{HI}}$ and $L_{B}$ in Haynes \& Giovanelli (1984). We have considered $h=H_{0} / 100=0.75$. We adapted their results, which were based on $\operatorname{mag}_{z w}$ to our use of $B_{\mathrm{c}}^{\mathrm{T}}$ with the relation found by Verdes-Montenegro et al. (2005) $\left(\operatorname{mag}_{z w}=B_{\mathrm{c}}^{\mathrm{T}}+0.136\right)$. Furthermore, considering that we express $L_{B}$ as a function of the solar bolometric luminosity ( $\left.\operatorname{mag}=4.75\right)$, we introduce the correction

$\left(\log L_{B}\right)_{\text {Haynes }}=\left(\log L_{B}\right)_{\text {ours }}+0.14$

to express $L_{B}$ in the terms we assume (Sect. 2) to calculate the expected content of HI. The deficiencies in $M_{\mathrm{H}_{2}}, L_{\mathrm{FIR}}$, and $M_{\mathrm{HI}}$ derived from $L_{B}$ are listed in Table 5.

\subsection{1. $M_{\mathrm{H}_{2}}$ and $L_{\mathrm{FIR}}$ deficiencies}

The mean $M_{\mathrm{H}_{2}}$ and $L_{\mathrm{FIR}}$ deficiencies for spiral galaxies in HCGs are similar, as can be seen in Table 6. Galaxies showing an excess in $M_{\mathrm{H}_{2}}$ or $L_{\mathrm{FIR}}$ have values spanning the full range of $L_{B}$, as can be seen in Fig. 4. Thus, the excess in $M_{\mathrm{H}_{2}}$ or $L_{\mathrm{FIR}}$ is not associated with the brightest objects per se. We checked in detail the properties of the nine galaxies showing the largest $M_{\mathrm{H}_{2}}$ excess (HCG 10c, HCG 16a, HCG 16c, HCG 16d, HCG 23b, HCG 23d, HCG 40c, HCG 58a, HCG 88c), and we find that half of them present strong signs of distortion (tidal tails in the optical and/or HI, kinematical perturbations, etc.).

Figure 8 (left) shows $\operatorname{Def}\left(M_{\mathrm{H}_{2}}\right)$ (from $\left.L_{B}\right)$ vs. $\operatorname{Def}\left(L_{\mathrm{FIR}}\right)$ for each galaxy. Both are strongly correlated, which can be understood coming from the causal relation between the molecular gas and SFR, leading to a lower SFR if the molecular gas as the fuel for SF decreases. For comparison, Fig. 8 (right) displays $\operatorname{Def}\left(M_{\mathrm{H}_{2}}\right)$ of the isolated galaxies versus their $\operatorname{Def}\left(L_{\mathrm{FIR}}\right)$. The behavior of the isolated galaxies does not show a significant difference compared to galaxies in HCGs with a very similar range covered by both samples. However, for the isolated galaxies, $\operatorname{Def}\left(M_{\mathrm{H}_{2}}\right)$ extends to slightly lower values for a given $\operatorname{Def}\left(L_{\mathrm{FIR}}\right)$. This is also reflected in the mean values of $\operatorname{Def}\left(M_{\mathrm{H}_{2}}\right)$ and $\operatorname{Def}\left(L_{\mathrm{FIR}}\right)$ of AMIGA and HCG galaxies (Table 6): (i) values of $\operatorname{Def}\left(L_{\text {FIR }}\right)$ for spiral galaxies are almost the same for both samples, and (ii) $\operatorname{Def}\left(M_{\mathrm{H}_{2}}\right)$ in spirals is higher by $0.15-0.20$ for HCG 
Table 6. Mean values and their errors for spiral galaxies $(T \geq 1)$ in HCGs and from the AMIGA sample.

\begin{tabular}{lcccc}
\hline \hline & \multicolumn{2}{c}{ HCGs } & \multicolumn{2}{c}{ AMIGA } \\
& Mean & $n_{\mathrm{UL}} / n^{1}$ & Mean & $n_{\mathrm{UL}} / n^{1}$ \\
\hline $\log \left(L_{B}\right)\left(L_{\odot}\right)$ & $9.95 \pm 0.06$ & $0 / 46$ & $9.75 \pm 0.04$ & $0 / 150$ \\
$\log \left(M_{\mathrm{H}_{2}}\right)\left(M_{\odot}\right)$ & $9.02 \pm 0.09$ & $11 / 46$ & $8.38 \pm 0.09$ & $64 / 150$ \\
$\log \left(L_{\mathrm{FIR}}\right)\left(L_{\odot}\right)$ & $9.53 \pm 0.09$ & $15 / 45$ & $9.16 \pm 0.05$ & $58 / 149$ \\
\hline $\operatorname{Def}\left(M_{\mathrm{H}_{2}}\right)\left(\right.$ from $\left.L_{B}\right)$ & $-0.14 \pm 0.09$ & $11 / 46$ & $0.06 \pm 0.04$ & $64 / 150$ \\
$\operatorname{Def}\left(M_{\mathrm{H}_{2}}\right)\left(\right.$ from $\left.L_{K}\right)$ & $-0.15 \pm 0.06$ & $10 / 45$ & $-0.01 \pm 0.05$ & $50 / 135$ \\
$\operatorname{Def}\left(L_{\mathrm{FIR}}\right)$ & $-0.11 \pm 0.08$ & $15 / 45$ & $-0.09 \pm 0.03$ & $58 / 149$ \\
$\operatorname{Def}(\mathrm{HI})$ & $0.93 \pm 0.13$ & $9 / 37$ & - & - \\
\hline $\log \left(M_{\mathrm{H}_{2}} / L_{B}\right)$, all $L_{B}$ & $-0.96 \pm 0.08$ & $11 / 46$ & $-1.25 \pm 0.04$ & $64 / 150$ \\
$\left(M_{\odot} / L_{\odot}\right)$ & & & & \\
$\log \left(M_{\mathrm{H}_{2}} / L_{B}\right), L_{B} \leq 10^{10} L_{\odot}$ & $-1.04 \pm 0.10$ & $10 / 22$ & $-1.36 \pm 0.05$ & $56 / 103$ \\
$\left(M_{\odot} / L_{\odot}\right)$ & & & & \\
$\log \left(M_{\mathrm{H}_{2}} / L_{B}\right), L_{B}>10^{10} L_{\odot}$ & $-0.88 \pm 0.09$ & $1 / 24$ & $-1.06 \pm 0.05$ & $8 / 47$ \\
$\left(M_{\odot} / L_{\odot}\right)$ & & & & \\
$\log \left(M_{\mathrm{H}_{2}} / L_{K}\right)$ & $-1.58 \pm 0.05$ & $10 / 45$ & $-1.76 \pm 0.05$ & $50 / 135$ \\
$\left(M_{\odot} / L_{K, \odot}\right)$ & & & & \\
$\log \left(L_{\mathrm{FIR}} / L_{B}\right)$ & $-0.45 \pm 0.07$ & $15 / 45$ & $-0.52 \pm 0.03$ & $58 / 149$ \\
\hline
\end{tabular}

Notes. ${ }^{(1)}$ For each subsample, $n$ is the number of galaxies and $n_{\mathrm{UL}}$ is the number of upper limits.

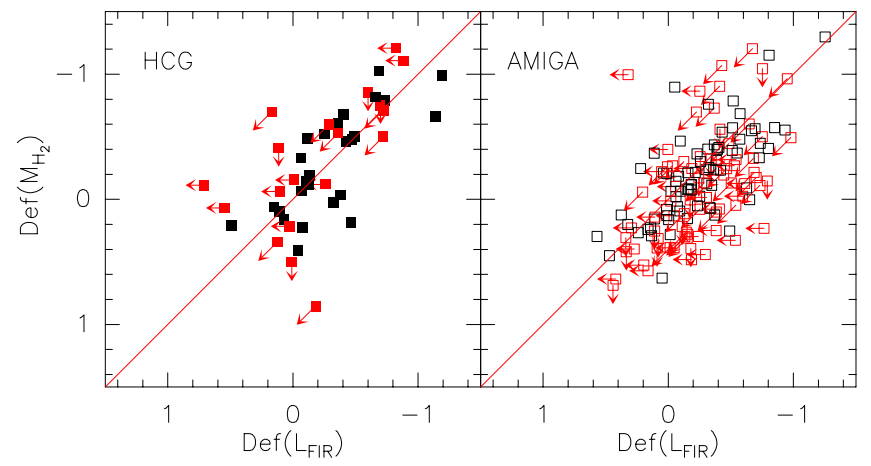

Fig. 8. $M_{\mathrm{H}_{2}}$ deficiency vs. $L_{\mathrm{FIR}}$ deficiency for late-type $(T \geq 1)$ galaxies in HCGs (left) and from the AMIGA sample (right). Red symbols represent upper limits in either $M_{\mathrm{H}_{2}}$ or $L_{\mathrm{FIR}}$, and black symbols detections. The $y=x$ line is plotted as reference and does not represent a fit to the data.

than for AMIGA galaxies (corresponding to a 40-60\% higher $M_{\mathrm{H}_{2}}$ than expected for isolated galaxies).

The histograms shown in Fig. 9 underline these findings: whereas the distribution of $\operatorname{Def}\left(M_{\mathrm{H}_{2}}\right)$ for spiral galaxies in HCGs is shifted to negative deficiencies (i.e. an excess) compared to AMIGA galaxies, the distribution of $\operatorname{Def}\left(L_{\mathrm{FIR}}\right)$ is very similar for spiral galaxies in HCGs and in the AMIGA sample. Two sample tests (Gehan's Generalized Wilcoxon Test and Logrank Test) confirm that the distributions of $\operatorname{Def}\left(M_{\mathrm{H}_{2}}\right)$ are different with a probability of $>96 \%$, whereas the distributions $\operatorname{Def}\left(L_{\mathrm{FIR}}\right)$ are identical with a non-negligible probability.

As an additional test, we compared the ratios $M_{\mathrm{H}_{2}} / L_{B}$ and $M_{\mathrm{H}_{2}} / L_{K}$ of HCG galaxies to those of isolated galaxies (listed in Table 6). In the case of $M_{\mathrm{H}_{2}} / L_{B}$ we derived the ratios both for the entire luminosity range and for low $\left(L_{B} \leq 10^{10} L_{\odot}\right)$ and high $\left(L_{B}>10^{10} L_{\odot}\right)$ luminosity galaxies in order not to be affected by the nonlinearity of the $M_{\mathrm{H}_{2}}-L_{B}$ relation. In all cases we found a lower ratio (by $\sim 0.2-0.3 \mathrm{dex}$ ) for the isolated galaxies, confirming our findings from the deficiency parameter.

The higher $M_{\mathrm{H}_{2}}$ for a given $L_{B}$ found for spiral galaxies in HGCs could be explained in three ways: a) a real excess of the total molecular gas mass (and will be further discussed as such in the following section); b) a higher concentration towards the
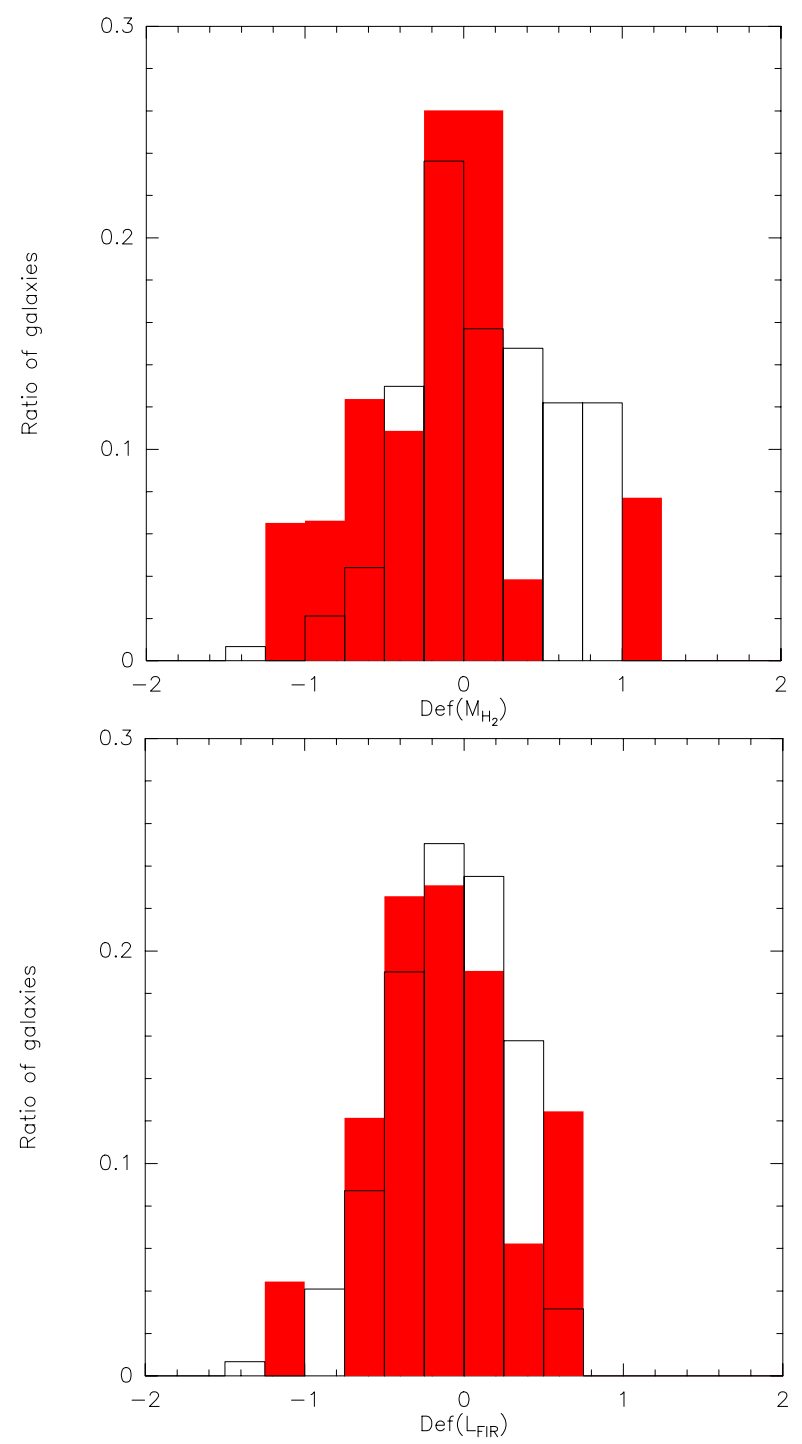

Fig. 9. $\operatorname{Def}\left(M_{\mathrm{H}_{2}}\right)$ (top) and $\operatorname{Def}\left(L_{\mathrm{FIR}}\right)$ (bottom) distribution of spiral galaxies in AMIGA (black line) and in HCGs (red filled bars), calculated with ASURV in order to take the upper limits into account. 
V. Martinez-Badenes et al.: Molecular gas content and SFR in Hickson compact groups

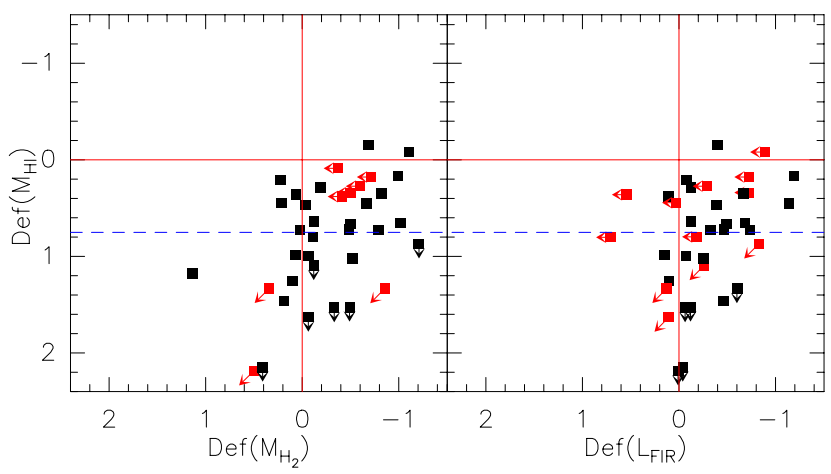

Fig. 10. $M_{\mathrm{HI}}$ vs. $M_{\mathrm{H}_{2}}$ deficiencies (left) and $M_{\mathrm{HI}}$ vs. $L_{\mathrm{FIR}}$ deficiencies (right) for spiral galaxies $(T \geq 1)$. The red lines show $\operatorname{Def}\left(M_{\mathrm{HI}}\right)=0, \operatorname{Def}\left(M_{\mathrm{H}_{2}}\right)=0$ and $\operatorname{Def}\left(L_{\mathrm{FIR}}\right)=0$, and the dashed lines give $\operatorname{Def}\left(M_{\mathrm{H}_{2}}\right)=0.75$, separating low and highly HI-deficient galaxies in our analysis. Red symbols denote upper limits in $M_{\mathrm{H}_{2}}$ or $L_{\mathrm{FIR}}$.

center of the molecular gas in HCG galaxies compared to isolated galaxies, so that the extrapolation of the flux based on a similar extent (see Sect. 3.1.3) would lead to an overestimate of $M_{\mathrm{H}_{2}}$, or c) a systematic difference in the CO-to- $\mathrm{H}_{2}$ conversion factor between the AMIGA and HCG sample. Although we cannot exclude this possibility, we do not consider it very likely. The CO-to- $\mathrm{H}_{2}$ conversion factor is known to depend on a number of galactic properties such as the metallicity, gas temperture, gas density, and velocity dispersion (e.g. Maloney \& Black 1988; Narayanan et al. 2011). These properties are likely similar in both samples because of the similar ranges in $L_{B}$ and $L_{\text {FIR }}$ (tracing SFR) that they cover. The first two effects ( $a$ and $b$ ) could both be at work at the same time. In fact, as indicated in e.g. Leon et al. (2008), galaxies in the AMIGA sample are dominated by disk SF while surveys of compact groups (Menon 1995) show that most radio detections involve compact nuclear emission. This can be explained since nuclear emission is thought to be enhanced by interactions that produce a loss of angular momentum of the molecular gas, which subsequently falls towards the center of the galaxy. These dissipative effects are likely near minimum in isolated galaxies. This result was also proposed by VerdesMontenegro et al. (1998), where the enhanced $I_{25} / I_{100}$ ratio in HCGs is said to be caused by local starbursts, presumably in the nuclear region. This result is still compatible with the conclusion of a normal level of FIR emission among the HCG galaxies that we find here, if the activity responsible for enhanced $24 \mu \mathrm{m}$ emission and enhanced/more concentrated molecular gas is localized compared to the overall distribution of gas and dust in the galaxies.

\subsubsection{Comparison to the $M_{\mathrm{HI}}$ deficiency}

In Fig. 10 we display $\operatorname{Def}\left(M_{\mathrm{HI}}\right)$ vs. $\operatorname{Def}\left(M_{\mathrm{H}_{2}}\right)$ (left) and $\operatorname{Def}\left(L_{\mathrm{FIR}}\right)$ (right). The mean value of $\operatorname{Def}\left(M_{\mathrm{HI}}\right)$ of the galaxies with available HI data is $0.93 \pm 0.13$ ( $12 \%$ of the expected value) for spiral galaxies and $1.31 \pm 0.11$ ( $5 \%$ of the expected value) for all morphological types, which is one order of magnitude more than $\operatorname{Def}\left(M_{\mathrm{H}_{2}}\right)$ and $\operatorname{Def}\left(L_{\mathrm{FIR}}\right)$. We stress here that the samples used in the present paper and in Verdes-Montenegro et al. (2001) are not the same. This earlier study concentrated on the set of data available at that time, which was biased towards HI bright groups. Later, more groups with higher HI deficiencies have been observed with the VLA (Verdes-Montenegro et al. 2007) and are part of the present sample. Therefore, the mean HI deficiency of the galaxies in Verdes-Montenegro et al. (2001) $(25 \%$ of the expected value for spiral galaxies) is less than the mean
HI deficiency of the present sample. We have checked that the $\mathrm{HI}$ deficiencies calculated in this paper are consistent with the values for the groups in common with Verdes-Montenegro et al. (2001).

Most noticeable in Fig. 10 is that even very HI-deficient galaxies have a fairly normal $M_{\mathrm{H}_{2}}$ or $L_{\mathrm{FIR}}$. There is no clear correlation between $\operatorname{Def}\left(M_{\mathrm{HI}}\right)$ and $\operatorname{Def}\left(M_{\mathrm{H}_{2}}\right)$ or $\operatorname{Def}\left(L_{\mathrm{FIR}}\right)$. There might be a weak trend in the sense that a higher $M_{\mathrm{HI}}$ deficiency leads to higher $M_{\mathrm{H}_{2}}$ and $L_{\mathrm{FIR}}$ deficiencies. This trend is also seen when calculating the mean deficiencies and ratios separately for low and highly $M_{\mathrm{HI}}$ deficient galaxies, here chosen as galaxies with $\operatorname{Def}\left(M_{\mathrm{HI}}\right)<0.75$ and $\operatorname{Def}\left(M_{\mathrm{HI}}\right)>0.75$ in order to obtain two groups of roughly the same size (Table 7). However, the differences are small and fall below significance when changing the separation to $\operatorname{Def}\left(M_{\mathrm{HI}}\right)=0.50$. Thus, the statistics in our sample are not sufficient to firmly conclude whether this trend is real.

\subsection{Comparison to the HI content and evolutionary stage of the group}

To study the influence of the global HI content of the group on $M_{\mathrm{H}_{2}}$ and SFR of the individual galaxies we have classified the groups as a function of their $\operatorname{Def}\left(M_{\mathrm{HI}}\right)$ as listed in Sect. 2. The average $\operatorname{Def}\left(M_{\mathrm{H}_{2}}\right)$ and $\operatorname{Def}\left(L_{\mathrm{FIR}}\right)$ of the galaxies belonging to these groups are given in Table 7 . We find no relation between the $\operatorname{Def}\left(M_{\mathrm{H}_{2}}\right)$ of the galaxies and the global $\operatorname{Def}\left(M_{\mathrm{HI}}\right)$ of the groups, and neither between the $\operatorname{Def}\left(L_{\mathrm{FIR}}\right)$ and the $\operatorname{Def}\left(M_{\mathrm{HI}}\right)$ of the groups.

In a similar way, we calculated the average $\operatorname{Def}\left(M_{\mathrm{H}_{2}}\right)$ and $\operatorname{Def}\left(L_{\mathrm{FIR}}\right)$ of the galaxies belonging to HCGs in different evolutionary states, as defined by (Borthakur et al. 2010, see Sect. 2), which are also given in Table 7 . The $\operatorname{Def}\left(M_{\mathrm{H}_{2}}\right)$ of the galaxies increases slightly as the group evolves along the evolutionary sequence. This trend is also visible in the ratios $M_{\mathrm{H}_{2}} / L_{B}$ and $M_{\mathrm{H}_{2}} / L_{K}$. For $\operatorname{Def}\left(L_{\mathrm{FIR}}\right)$, there is no clear relation for spiral galaxies with the evolutionary state, and we only find a trend when considering the total sample, most likely due to a changing fraction of ellipticals.

A very pronounced variation with evolutionary phase is shown by the morphological types (Fig. 11). The ratio of elliptical and S0 galaxies increase strongly in groups in phase 3. It has been proposed (e.g. Verdes-Montenegro et al. 2001; Bekki \& Couch 2011) that S0 galaxies in HCGs might be stripped spirals.

\subsection{Star formation rate, star formation efficiency and specific SFR}

We calculate the SFR from $L_{\text {FIR }}$ following the prescription of Kennicutt (1998):

$\operatorname{SFR}\left(M_{\odot} / \mathrm{yr}\right)=4.5 \times 10^{-44} L_{\mathrm{IR}}\left(\mathrm{erg} \mathrm{s}^{-1}\right)$

where $L_{\mathrm{IR}}$ refers to the IR luminosity integrated over the entire mid- and far-IR spectrum $(10-1000 \mu \mathrm{m})$. This expression is based on a Salpeter initial mass function (IMF). We convert it to the Kroupa (2001) IMF by dividing by a factor 1.59 (Leroy et al. 2008). In our analysis we use $L_{\text {FIR }}$ (Eq. (6)), which estimates the FIR emission in the wavelength range of $42.5-122.5 \mu \mathrm{m}$. We estimate $L_{\mathrm{IR}}$ from $L_{\mathrm{FIR}}$ using the result of Bell (2003) that on average $L_{\mathrm{IR}} \sim 2 \times L_{\mathrm{FIR}}$. Taking this into account, we can calculate the SFR from $L_{\mathrm{FIR}}$ as

$$
\begin{aligned}
\operatorname{SFR}\left(M_{\odot} / \mathrm{yr}\right) & =4.5 \times 2 \times \frac{1}{1.59} \times 10^{-44} L_{\mathrm{FIR}}\left(\mathrm{erg} \mathrm{s}^{-1}\right) \\
& =2.2 \times 10^{-10} L_{\mathrm{FIR}}\left(L_{\odot}\right) .
\end{aligned}
$$


Table 7. Mean values and their errors of deficiencies and ratios of $M_{\mathrm{H}_{2}}$ and $L_{\mathrm{FIR}}$ for different samples, considering only spiral galaxies ( $\left.T \geq 1\right)$.

\begin{tabular}{|c|c|c|c|c|c|c|}
\hline & & $\operatorname{Def}\left(M_{\mathrm{H}_{2}}\right)$ & $\begin{array}{c}\log \left(M_{\mathrm{H}_{2}} / L_{B}\right) \\
\left(M_{\odot} / L_{\odot}\right)\end{array}$ & $n_{\mathrm{UL}} / n$ & $\begin{array}{c}\log \left(M_{\mathrm{H}_{2}} / L_{K}\right) \\
\left(M_{\odot} / L_{K, \odot}\right)\end{array}$ & $n_{\mathrm{UL}} / n$ \\
\hline Total & & $-0.14 \pm 0.09$ & $-0.96 \pm 0.08$ & $11 / 46$ & $-1.58 \pm 0.05$ & $10 / 45$ \\
\hline HI content & $\operatorname{Def}(\mathrm{HI})<0.75$ & $-0.34 \pm 0.10$ & $-0.82 \pm 0.10$ & $5 / 21$ & $-1.40 \pm 0.07$ & $4 / 20$ \\
\hline of galaxies & $\operatorname{Def}(\mathrm{HI})>0.75$ & $-0.07 \pm 0.16$ & $-1.15 \pm 0.13$ & $3 / 16$ & $-1.77 \pm 0.08$ & $3 / 16$ \\
\hline HI content & Normal & $-0.38 \pm 0.20$ & $-0.76 \pm 0.15$ & $1 / 6$ & $-1.52 \pm 0.11$ & $0 / 5$ \\
\hline \multirow[t]{2}{*}{ of the group } & Slightly deficient & $-0.08 \pm 0.11$ & $-0.99 \pm 0.10$ & $8 / 32$ & $-1.59 \pm 0.07$ & $8 / 32$ \\
\hline & Very deficient & $-0.21 \pm 0.08$ & $-0.95 \pm 0.10$ & $2 / 8$ & $-1.60 \pm 0.12$ & $2 / 8$ \\
\hline Evolutionary & Phase 1 & $-0.35 \pm 0.14$ & $-0.76 \pm 0.11$ & $2 / 11$ & $-1.46 \pm 0.09$ & $1 / 10$ \\
\hline \multirow[t]{3}{*}{ Phase } & Phase 2 & $-0.16 \pm 0.13$ & $-0.92 \pm 0.12$ & $5 / 21$ & $-1.55 \pm 0.07$ & $5 / 21$ \\
\hline & Phase 3 & $-0.04 \pm 0.09$ & $-1.07 \pm 0.08$ & $4 / 14$ & $-1.71 \pm 0.11$ & $4 / 14$ \\
\hline & & $\overline{\operatorname{Def}\left(L_{\mathrm{FIR}}\right)}$ & $\log \left(L_{\mathrm{FIR}} / L_{B}\right)$ & $n_{\mathrm{UL}} / n$ & $\begin{array}{c}\log \left(L_{\mathrm{FIR}} / L_{K}\right) \\
\left(L_{\odot} / L_{K \odot \odot}\right)\end{array}$ & $n_{\mathrm{UL}} / n$ \\
\hline Total & & $-0.11 \pm 0.08$ & $-0.45 \pm 0.07$ & $15 / 45$ & $-1.14 \pm 0.09$ & $14 / 44$ \\
\hline HI content & $\operatorname{Def}(\mathrm{HI})<0.75$ & $-0.32 \pm 0.11$ & $-0.28 \pm 0.11$ & $6 / 20$ & $-0.84 \pm 0.10$ & $5 / 19$ \\
\hline of galaxies & $\operatorname{Def}(\mathrm{HI})>0.75$ & $0.03 \pm 0.11$ & $-0.60 \pm 0.11$ & $6 / 16$ & $-1.38 \pm 0.13$ & $6 / 16$ \\
\hline HI content & Normal & $-0.19 \pm 0.19$ & $-0.36 \pm 0.11$ & $3 / 6$ & $-1.07 \pm 0.17$ & $2 / 5$ \\
\hline \multirow[t]{2}{*}{ of the group } & Slightly deficient & $-0.08 \pm 0.09$ & $-0.45 \pm 0.09$ & $10 / 31$ & $-1.15 \pm 0.11$ & $10 / 31$ \\
\hline & Very deficient & $-0.23 \pm 0.08$ & $-0.37 \pm 0.07$ & $2 / 8$ & $-1.03 \pm 0.14$ & $2 / 8$ \\
\hline Evolutionary & Phase 1 & $-0.17 \pm 0.15$ & $-0.36 \pm 0.13$ & $4 / 11$ & $-1.03 \pm 0.13$ & $3 / 10$ \\
\hline \multirow[t]{2}{*}{ Phase } & Phase 2 & $-0.12 \pm 0.13$ & $-0.43 \pm 0.13$ & $7 / 20$ & $-1.11 \pm 0.14$ & $7 / 20$ \\
\hline & Phase 3 & $-0.12 \pm 0.05$ & $-0.45 \pm 0.04$ & $4 / 14$ & $-1.11 \pm 0.09$ & $4 / 14$ \\
\hline
\end{tabular}

Notes. $n$ is the number of galaxies and $n_{\mathrm{UL}}$ is the number of upper limits.

Table 8. Mean $\log (\mathrm{SFE})$ for different samples and measurements (only spiral galaxies, $T \geq 1)$.

\begin{tabular}{lc}
\hline \hline Sample & $\left\langle\log (\mathrm{SFE})\left(\mathrm{yr}^{-1}\right)\right\rangle$ \\
\hline HCGs & $-9.06 \pm 0.05^{1} /-9.22 \pm 0.06^{2}$ \\
CIGs & $-8.94 \pm 0.03^{1} /-9.07 \pm 0.04^{2}$ \\
HERACLES & $-9.23^{3}$ \\
\hline
\end{tabular}

Notes. ${ }^{(1)}$ Galaxies detected in both $\mathrm{CO}$ and FIR and galaxies detected in FIR but not in CO. ${ }^{(2)}$ Galaxies detected in both $\mathrm{CO}$ and FIR and galaxies detected in CO but not in FIR. ${ }^{(3)}$ From Bigiel et al. (2011). The $1 \sigma$ standard deviation is 0.24 dex.

The values of the SFR of the galaxies in our sample are listed in Table 3.

We define the SFE as the ratio between the SFR and the molecular gas mass, $\mathrm{SFE}=S F R / M_{\mathrm{H}_{2}}$. The SFE of the individual galaxies in our sample are listed in Table 3. Figure 6 shows a good, roughly linear correlation between $L_{\mathrm{FIR}}$ and $M_{\mathrm{H}_{2}}$ and indicates that the SFE in our sample is expected to show a fairly narrow range. To calculate the average SFE of our sample, we must consider that ASURV can only handle data showing upper or lower limits, but not both. Thus, we first calculated the average SFE considering only galaxies detected in $\mathrm{CO}$ with an upper limit in FIR, together with the ones detected in both bands. Separately, we considered only those detected in FIR but not detected in $\mathrm{CO}$ and the ones detected in both bands. The average values are listed in Table 8 .

We calculated the average SFE for the AMIGA sample of isolated galaxies, taking $M_{\mathrm{H}_{2}}$ from Lisenfeld et al. (2011) and $L_{\text {FIR }}$ from Lisenfeld et al. (2007), for comparison with the SFE in HCG galaxies. The values are listed in Table 8. We furthermore list the SFE derived from a spatially resolved analysis for 30 nearby galaxies from the HERACLES survey (Bigiel et al. 2011). All values are adjusted to our $\mathrm{CO}-$ to- $\mathrm{H}_{2}$ conversion factor, Kroupa IMF, and no consideration of helium in the molecular gas mass. Table 8 shows a slightly lower SFE in HCGs than in AMIGA galaxies, in line with the previous results of an excess in $M_{\mathrm{H}_{2}}$ but a normal value of $L_{\mathrm{FIR}}$. In comparison to the galaxies from the HERACLES survey there is no noticeable difference. Thus, overall there are no strong indications that the process of SF occurs in a different manner in the different environments of HCGs.

The specific SFR, sSFR, is defined as the ratio between the SFR and the stellar mass of a galaxy. We calculated the stellar mass from the $K$ band luminosity since the light in this band is dominated by the emission of low-mass stars, which are responsible for the bulk of stellar mass in galaxies. From $L_{K}$ we derived the stellar mass, $M_{*}$, by adopting a mass-to-luminosity ratio of $M_{\odot} / L_{K, \odot}=1.32$ (Cole et al. 2001) for the Salpeter IMF, and applying a correction factor of 0.5 (from Bell et al. 2003) to change to the Kroupa (2001) IMF used in this paper. The values for the individual galaxies are listed in Table 3 . The average sSFR for spiral galaxies in our sample is $\log (\mathrm{s} S F R)=-10.61 \pm 0.07 \mathrm{yr}^{-1}$.

\subsubsection{SFE and sSFR functions of the deficiencies of the galaxies}

In Fig. 12 we display the SFE and the sSFR of the spiral galaxies in our sample as a function of their $\operatorname{Def}\left(M_{\mathrm{HI}}\right)$ and $\operatorname{Def}\left(M_{\mathrm{H}_{2}}\right)$. There is no clear trend of the SFE with the gas deficiency of the galaxies, whether atomic or molecular. This is confirmed by the mean values listed in Table 9. This result indicates that SF proceeds with the same efficiency, regardless of whether it occurs in a galaxy with a low or high $M_{\mathrm{HI}}$ deficiency.

On the other hand, galaxies with lower $\operatorname{Def}\left(M_{\mathrm{H}_{2}}\right)$ or $\operatorname{Def}\left(M_{\mathrm{HI}}\right)$ tend to have a higher sSFR (see Fig.12, as well as Table 9 for the quantitative trends). In particular, the trend with $\operatorname{Def}\left(M_{\mathrm{HI}}\right)$ is interesting because it suggests that, although the $\operatorname{Def}\left(M_{\mathrm{HI}}\right)$ of a galaxy has no influence on the absolute SFR or SFE, it has a noticeable effect on the SFR per stellar mass.

\subsection{Line ratio}

Figure 13 shows the $\mathrm{CO}(1-0)$ versus the $\mathrm{CO}(2-1)$ intensity for the galaxies we observed (Sect. 3.1.1). The plotted intensities 
V. Martinez-Badenes et al.: Molecular gas content and SFR in Hickson compact groups

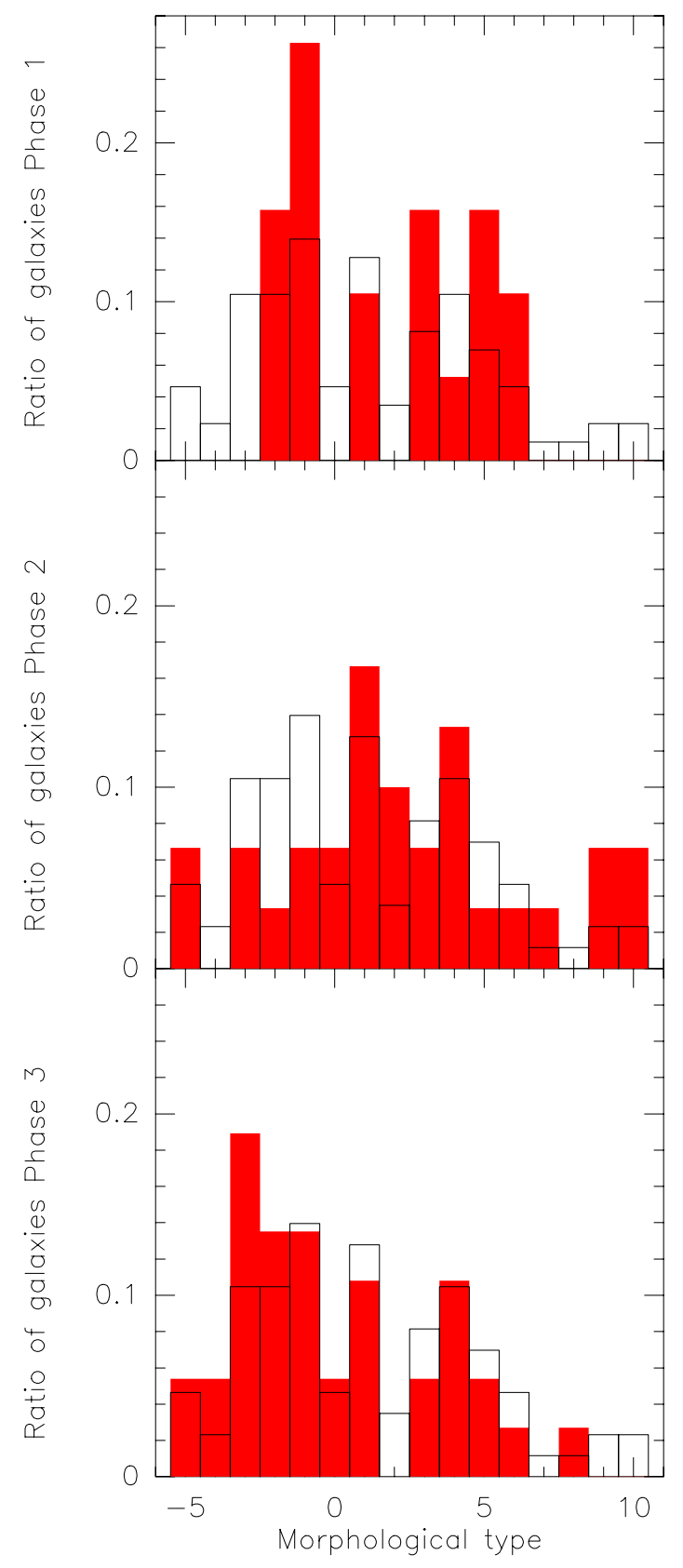

Fig. 11. Morphological type distribution for different evolutionary phases. From top to bottom, the morphological type distribution of galaxies in HCGs in evolutionary phases 1, 2, and 3 are plotted. The filled red bins correspond to the distribution for the groups in each evolutionary state, while the black line bins correspond, for comparison, to galaxies of all phases.

are not aperture corrected. The mean ratio between both intensities is $I_{\mathrm{CO}(2-1)} / I_{\mathrm{CO}(1-0)}=1.13 \pm 0.11$ for the full sample and $1.13 \pm 0.12$ for spiral galaxies alone. To calculate this mean ratio with ASURV, we have taken galaxies into account with detections in both CO transitions, as well as those detected only in $\mathrm{CO}(1-0)$. These values are slightly higher than those found by Leroy et al. (2009) from $\mathrm{CO}(2-1)$ and $\mathrm{CO}(1-0)$ maps for nearby galaxies from the SINGS sample $\left(I_{\mathrm{CO}(2-1)} / I_{\mathrm{CO}(1-0)} \sim 0.8\right)$ and than those from Braine et al. (1993) who obtained a mean line ratio of $I_{\mathrm{CO}(2-1)} / I_{\mathrm{CO}(1-0)}=0.89 \pm 0.06$ for a sample of nearby spiral galaxies. Both values are, in contrast to ours, corrected for beam-size effects.
Table 9. Mean $\log (\mathrm{sSFR})$ and $\log (\mathrm{SFE})$ as a function of $\operatorname{Def}\left(M_{\mathrm{HI}}\right)$ and $\operatorname{Def}\left(M_{\mathrm{H}_{2}}\right)$ for spiral galaxies $(T \geq 1)$.

\begin{tabular}{|c|c|c|}
\hline & \multicolumn{2}{|c|}{$\log (\mathrm{sSFR})\left(\mathrm{yr}^{-1}\right)$} \\
\hline & Mean & $n_{\mathrm{UL}} / n$ \\
\hline $\operatorname{Def}\left(M_{\mathrm{HI}}\right)<0.75$ & $-10.31 \pm 0.10$ & $(5 / 19)$ \\
\hline $\operatorname{Def}\left(M_{\mathrm{HI}}\right)>0.75$ & $-10.85 \pm 0.13$ & $(6 / 16)$ \\
\hline $\operatorname{Def}\left(M_{\mathrm{H}_{2}}\right)<-0.25$ & $-10.33 \pm 0.07$ & $(6 / 22)$ \\
\hline \multirow[t]{3}{*}{$\operatorname{Def}\left(M_{\mathrm{H}_{2}}\right)>-0.25$} & $-10.81 \pm 0.12$ & $(8 / 22)$ \\
\hline & \multicolumn{2}{|c|}{$\log (\mathrm{SFE})\left(\mathrm{yr}^{-1}\right)$} \\
\hline & Mean & $n_{\mathrm{UL}} / n$ \\
\hline $\operatorname{Def}\left(M_{\mathrm{HI}}\right)<0.75$ & $-9.08 \pm 0.07$ & $(5 / 19)$ \\
\hline $\operatorname{Def}\left(M_{\mathrm{HI}}\right)>0.75$ & $-9.16 \pm 0.12$ & $(6 / 16)$ \\
\hline $\operatorname{Def}\left(M_{\mathrm{H}_{2}}\right)<-0.25$ & $-9.05 \pm 0.07$ & $(6 / 22)$ \\
\hline $\operatorname{Def}\left(M_{\mathrm{H}_{2}}\right)>-0.25$ & $-9.04 \pm 0.13$ & $(8 / 22)$ \\
\hline
\end{tabular}

Notes. $n$ is the number of galaxies and $n_{\mathrm{UL}}$ is the number of upper limits.

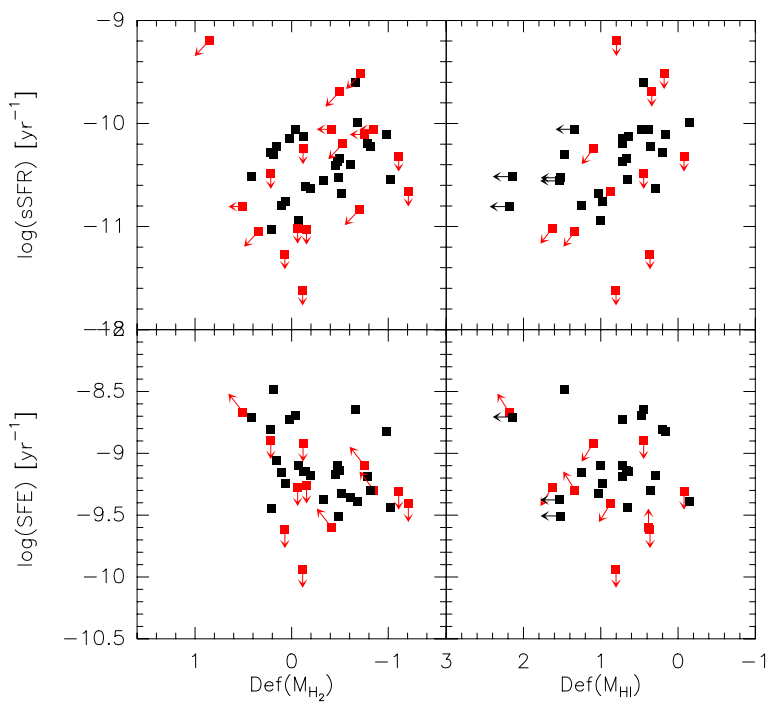

Fig. 12. Specific SFR (sSFR) (top) and star formation efficiency (SFE) (bottom), vs. $M_{\mathrm{H}_{2}}$ and $M_{\mathrm{HI}}$ deficiencies of spiral galaxies $(T \geq 1)$ in HCGs. Red symbols denote upper limits in $M_{\mathrm{H}_{2}}$ or $L_{\mathrm{FIR}}$.

To interpret the ratio of $I_{\mathrm{CO}(2-1)} / I_{\mathrm{CO}(1-0)}$ one has to consider two main parameters: the source distribution and the opacity. For optically thick, thermalized emission with a point-like distribution we expect a ratio $I_{\mathrm{CO}(2-1)} / I_{\mathrm{CO}(1-0)}=\left(\theta_{\mathrm{CO}(1-0)} / \theta_{\mathrm{CO}(2-1)}\right)^{2}=4$ (with $I_{\mathrm{CO}}$ in $T_{\mathrm{mb}}$ and $\theta$ being the FWHM of the beams). On the other hand, for a uniform source brightness distribution we expect ratios over 1 for optically thin gas, and ratios between about 0.6 and 1 for optically thick gas (with excitation temperatures above $5 \mathrm{~K}$ ).

Owing to the different beam sizes of $\mathrm{CO}(1-0)$ and $\mathrm{CO}(2-1)$ in our observations we cannot distinguish these two cases. However, we can conclude that our values are consistent with optically thick, thermalized gas with an extended distribution. Our mean value is slightly higher than the (beam-corrected) values of Leroy et al. (2009) and Braine et al. (1993) which might indicate that the molecular gas is not completely uniform over the $\mathrm{CO}(1-0)$ beam, but slightly concentrated towards the center.

\section{A possible evolutionary sequence of the molecular gas content and SFR in HCGs}

In contrast to the HI content, which can be highly deficient, the mean deficiencies for both $M_{\mathrm{H}_{2}}$ and $L_{\mathrm{FIR}}$ are low and close to the 


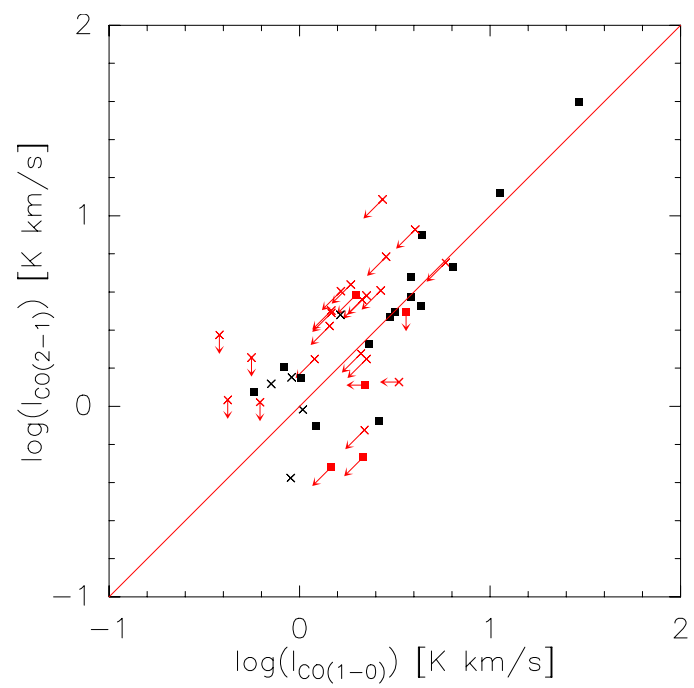

Fig. 13. $\log \left(I_{\mathrm{CO}(2-1)}\right)$ versus $\log \left(I_{\mathrm{CO}(1-0)}\right)$ for the galaxies observed by us. Spiral galaxies $(T \geq 1)$ are shown as filled squares and early-type $(T \leq 0)$ as crosses. Red symbols indicate upper limits in either $I_{\mathrm{CO}(2-1)}$ or $I_{\mathrm{CO}(1-0)}$, and black symbols are detections. The $y=x$ line is plotted as reference and does not represent a fit to the data.

values found for isolated galaxies. In the case of $M_{\mathrm{H}_{2}}$ we even find indications for a 40-60\% excess compared to isolated galaxies. The difference in deficiency between the atomic and molecular gas is most likely due to the larger extent of the HI gas, which can thus be removed more efficiently from the galaxies, while the molecular gas, which is typically more concentrated in the inner regions, is presumably less affected by the environment. Subsequently, the lower HI mass might cause lower $M_{\mathrm{H}_{2}}$, which in turn leads to lower SFR. It is, however, remarkable that galaxies with a high $\mathrm{HI}$ deficiency can still contain a considerable amount of molecular gas and continue to form stars with a normal SFE. This SF is not expected to last very long because once the molecular gas is used up, no $\mathrm{HI}$ is available to provide fuel for future SF.

Within this general picture of the relative normality of $M_{\mathrm{H}_{2}}$ and $L_{\mathrm{FIR}}$, we have found a relation between $\operatorname{Def}\left(M_{\mathrm{HI}}\right)$ and the sSFR, and a tentative trend with $\operatorname{Def}\left(M_{\mathrm{H}_{2}}\right)$ and $\operatorname{Def}\left(L_{\mathrm{FIR}}\right)$. Furthermore, there is a trend of $\operatorname{Def}\left(M_{\mathrm{H}_{2}}\right)$ with the evolutionary phase (Table 7), in the sense that galaxies in HCGs belonging to phase 1 have the highest excess in $M_{\mathrm{H}_{2}}$. These trends might suggest that two mechanisms are in play. First, an increasing $M_{\mathrm{HI}}$ deficiency can be interpreted within a scenario in which galaxies in HCGs lose part of their $\mathrm{HI}$ as a result of mostly tidal stripping during the initial evolutionary phase, as suggested in the evolutionary model of Verdes-Montenegro et al. (2001). On the other hand, in an early evolutionary phase the HI-to- $\mathrm{H}_{2}$ conversion rate might be enhanced as a result of the continuous interactions between galaxies, leading to the enhancement in $M_{\mathrm{H}_{2}}$ that we observe in evolutionary phase 1 . This enhancement of $M_{\mathrm{H}_{2}}$ cannot explain the high HI deficiencies observed in most galaxies, in agreement with the conclusions of Rasmussen et al. (2008), but it could partly explain the lack of HI, especially in the galaxies with the lowest HI deficiencies.

Based on our results, we thus suggest the following scenario, which is speculative but compatible with our observations. Galaxies in an HCG start with a normal content in $M_{\mathrm{H}_{2}}$ and $M_{\mathrm{HI}}$; i.e., they have $\operatorname{Def}\left(M_{\mathrm{HI}}\right)=0$ and $\operatorname{Def}\left(M_{\mathrm{H}_{2}}\right)=0$. Then, during the early evolutionary phase, tidal interactions enhance the conversion from atomic to molecular gas at the same time as they strip the $\mathrm{HI}$ from the galaxies, which leads to $\operatorname{Def}\left(M_{\mathrm{HI}}\right)>0$ and $\operatorname{Def}\left(M_{\mathrm{H}_{2}}\right)<0$. Finally, the multiple interactions within the group strip the main part of the $\mathrm{HI}$ in the disks, resulting in $\operatorname{Def}\left(M_{\mathrm{HI}}\right)>>0$ and, as a consequence also increase in $\operatorname{Def}\left(M_{\mathrm{H}_{2}}\right)$. This last effect could have contributed to an increase in the fraction of lenticular galaxies along the evolutionary sequence due to $\mathrm{HI}$ and $\mathrm{H}_{2}$ stripping of spirals.

\section{Summary and conclusions}

We analyzed data for $M_{\mathrm{H}_{2}}$, which was obtained from observations with the IRAM 30 m telescope and from the literature, $L_{\mathrm{FIR}}$ from IRAS, and $M_{\mathrm{HI}}$ for 86 galaxies in $20 \mathrm{HCGs}$ in order to study the relation between atomic gas, molecular gas, and SFR, traced by $L_{\text {FIR }}$ in these galaxies. We compared these properties to those of isolated galaxies from the AMIGA project (VerdesMontenegro et al. 2005). We adopted the same CO-to- $\mathrm{H}_{2}$ conversion factor for both samples. The main conclusions of our study can be summarized as follows:

- The relation between $M_{\mathrm{H}_{2}}, L_{\mathrm{FIR}}$, and $L_{B}$ in the galaxies in HCGs is not significantly different from the one found in isolated galaxies. The values of $L_{\mathrm{FIR}}$ for spirals galaxies in HCGs are similar to those of the AMIGA galaxies for the same $L_{B}$. For $M_{\mathrm{H}_{2}}$ we find, however, a slight, but statistically significant, excess $(\sim 50 \%)$ of HCGs spiral galaxies relative to AMIGA galaxies. This could alternatively be explained by a higher radial concentration of the molecular gas in HCG galaxies to the center when compared with isolated galaxies, so that the extrapolation of the flux based on a similar extent (see Sect. 3.1.3) would lead to overestimating $M_{\mathrm{H}_{2}}$ for the group galaxies. Another possible explanation could be a systematically lower $\mathrm{CO}-$ to- $\mathrm{H}_{2}$ conversion factor for spirals in HCGs.

- For elliptical and S0 galaxies, the large number of upper limits do not allow strong conclusions about their $M_{\mathrm{H}_{2}}$ or $L_{\mathrm{FIR}}$. We note, however that, while the $L_{B}$ range for S0s is comparable to isolated $\mathrm{S} 0$ galaxies, Es in HCGs are up to half an order magnitude more luminous in $L_{B}$ than isolated Es are.

- Practically all of our galaxies lie in the range of $L_{\mathrm{FIR}} / M_{\mathrm{H}_{2}}=1-10 L_{\odot} / M_{\odot}$, which is typical of normal, quiescent galaxies. The deficiencies in $M_{\mathrm{H}_{2}}$ and $L_{\mathrm{FIR}}$ are tightly correlated and cover about the same range as in isolated galaxies.

- The $M_{\mathrm{HI}}$ deficiency, calculated from the VLA data for individual galaxies, is much greater than the other deficiencies with a mean value of $0.93 \pm 0.13(12 \%$ of the expected value) for spiral galaxies and $1.31 \pm 0.11$ (5\% the expected value) for all morphological types, and it represents the largest difference with respect to isolated galaxies. These values are significantly higher than those obtained in VerdesMontenegro et al. (2001) since the sample in that study was biased towards HI bright galaxies, while here we present a redshift-selected sample.

- The SFE of the spiral galaxies in the HCGs is slightly lower than in isolated galaxies, but in the range of values found for nearby spiral galaxies (Bigiel et al. 2011). We have found no relation of the SFE with either $\operatorname{Def}\left(M_{\mathrm{HI}}\right)$ or $\operatorname{Def}\left(M_{\mathrm{H}_{2}}\right)$.

- There is a trend for the SSFR to increase with decreasing $\operatorname{Def}\left(M_{\mathrm{HI}}\right)$ and $\operatorname{Def}\left(M_{\mathrm{H}_{2}}\right)$. This suggests that, although the $\operatorname{Def}\left(M_{\mathrm{HI}}\right)$ of a galaxy only has a weak influence on the absolute SFR, it has a stronger influence on the SFR per stellar mass. 
V. Martinez-Badenes et al.: Molecular gas content and SFR in Hickson compact groups

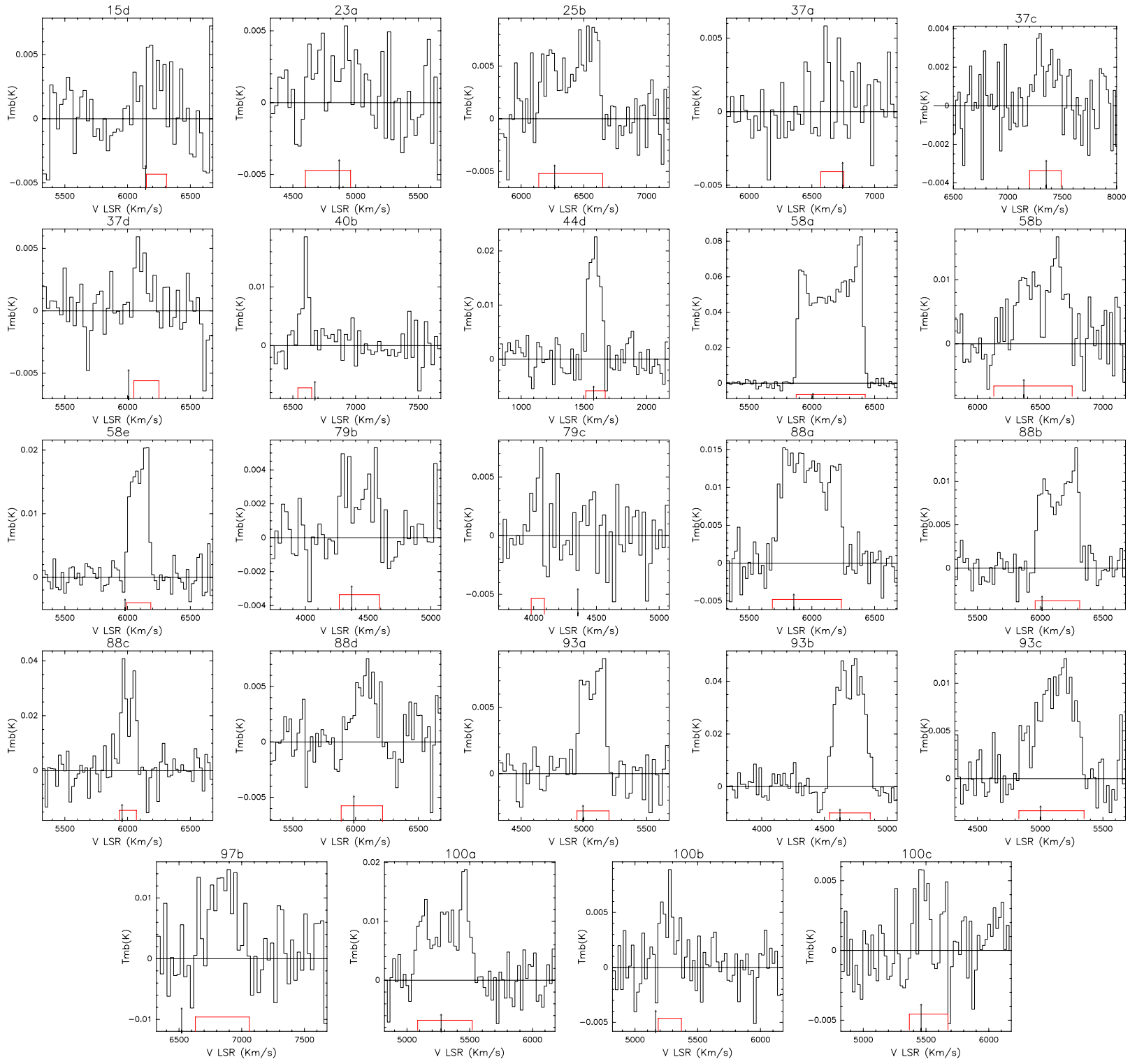

Fig. A.1. $\mathrm{CO}(1-0)$ spectra for the detected HCG galaxies. The detection window is shown with a red horizontal line. Main beam temperature $\left(T_{\mathrm{mb}}\right.$, in $\mathrm{K}$ ) is displayed on the $Y$ axis, and the velocity with respect to LSR in $\mathrm{km} \mathrm{s}^{-1}$ on the $X$ axis. Velocity resolution is smoothed to 21 or $27 \mathrm{~km} \mathrm{~s}^{-1}$. The optical velocity of the galaxy, converted to the radio definition, is marked with an arrow.

- There is a trend toward decreasing molecular gas deficiency with evolutionary phase, with galaxies in groups in an early phase showing an excess in $M_{\mathrm{H}_{2}}$. This excess goes to 0 in later phases. A similar trend might exist with $\operatorname{Def}\left(M_{\mathrm{HI}}\right)$, but is statistically only marginally significant in our sample. This is interpreted as an initial enhancement of the conversion from atomic to molecular gas due to ongoing tidal interactions, later followed by stripping of most of their HI. In these later phases, evolution of spiral to lenticular galaxies would explain both the overabundance of those morphological types and the $M_{\mathrm{HI}}$ deficiency and decrease in $M_{\mathrm{H}_{2}}$ content of the galaxies.

- No trend in the global HI deficiency of the groups is found, which suggests that the molecular gas content and SF are more driven by one-to-one interaction than directly by the local environment.

Acknowledgements. This work has been supported by the research projects AYA2008-06181-C02 and AYA2007-67625-C02-02 from the Spanish Ministerio de Ciencia y Educación and the Junta de Andalucía (Spain) grants
P08-FQM-4205, FQM-0108 and TIC-114. D.E. was supported by a Marie Curie International Fellowship within the 6th European Community Framework Program (MOIF-CT-2006-40298). U.L. warmly thanks IPAC (Caltech) for their hospitality during a sabbatical stay when this work was finished. We also thank T. Bitsakis and V. Charmandaris for letting us use their Spitzer data prior to publication, and the anonymous referee for critical comments that helped to put our conclusions on a firmer ground and improved the quality of the paper. This work is based on observations with the Instituto de Radioastronomia Milimétrica IRAM $30 \mathrm{~m}$ and the Five College Radio Astronomy (FCRAO) $14 \mathrm{~m}$. The FCRAO is supported by NSF grant AST 0838222. This research has made use of the NASA/IPAC Extragalactic Database (NED), which is operated by the Jet Propulsion Laboratory, California Institute of Technology, under contract with the National Aeronautics and Space Administration. We also acknowledge the usage of the HyperLeda database (http://leda.univ-lyon1.fr).

\section{Appendix A: CO spectra}

Figure A.1 shows the $\mathrm{CO}(1-0)$ spectra of the detections and tentative detections observed by us and Fig. A.1 the $\mathrm{CO}(2-1)$ spectra. 

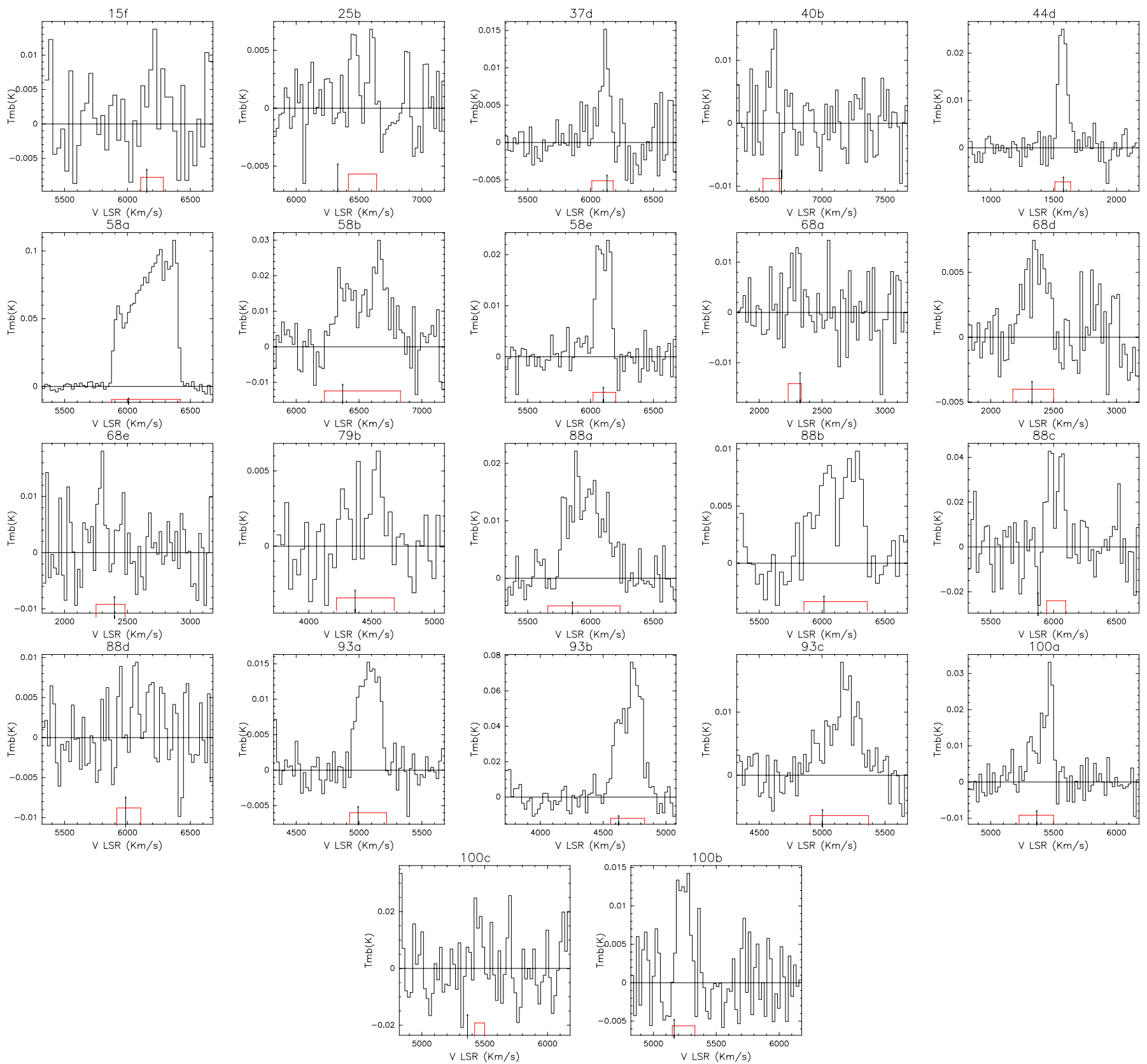

Fig. A.2. $\mathrm{CO}(2-1)$ spectra for the detected HCG galaxies. The detection window is shown with a red horizontal line. Main beam temperature $\left(T_{\mathrm{mb}}\right.$, in $\mathrm{K}$ ) is displayed on the $Y$ axis, and the velocity with respect to LSR in $\mathrm{km} \mathrm{s}^{-1}$ on the $X$ axis. Velocity resolution is smoothed to 21 or $27 \mathrm{~km} \mathrm{~s}^{-1}$. The optical velocity of the galaxy, converted to the radio definition, is marked with an arrow.

\section{References}

Bekki, K., \& Couch, W. J. 2011, MNRAS, 415, 1783

Bell, E. F. 2003, ApJ, 586, 794

Bell, E. F., McIntosh, D. H., Katz, N., \& Weinberg, M. D. 2003, ApJS, 149, 289

Bigiel, F., Leroy, A. K., Walter, F., et al. 2011, ApJ, 730, L13

Bitsakis, T., Charmandaris, V., Le Floc'h, E., et al. 2010, A\&A, 517, A75

Bitsakis, T., Charmandaris, V., da Cunha, E., et al. 2011, A\&A, 533, A142

Borthakur, S., Yun, M. S., \& Verdes-Montenegro, L. 2010, ApJ, 710, 385 Boselli, A., Mendes de Oliveira, C., Balkowski, C., Cayatte, V., \& Casoli, F. 1996, A\&A, 314, 738

Boselli, A., Gavazzi, G., Lequeux, J., et al. 1997, A\&A, 327, 522

Boselli, A., Lequeux, J., \& Gavazzi, G. 2002, A\&A, 384, 33

Braine, J., Combes, F., Casoli, F., et al. 1993, A\&AS, 97, 887

Calzetti, D., Wu, S., Hong, S., et al. 2010, ApJ, 714, 1256

Casasola, V., Bettoni, D., \& Galletta, G. 2004, A\&A, 422, 941

Casoli, F., Boisse, P., Combes, F., \& Dupraz, C. 1991, A\&A, 249, 359
Cole, S., Norberg, P., Baugh, C. M., et al. 2001, MNRAS, 326, 255

de Vaucouleurs, G., de Vaucouleurs, A., Corwin, Jr., H. G., et al. 1991, Third Reference Catalogue of Bright Galaxies (Springer)

Dickman, R. L., Snell, R. L., \& Schloerb, F. P. 1986, ApJ, 309, 326

Durbala, A., del Olmo, A., Yun, M. S., et al. 2008, AJ, 135, 130

Feigelson, E. D., \& Nelson, P. I. 1985, ApJ, 293, 192

Fernández-Lorenzo, M., Sulentic, J., \& Verdes-Montenegro, L. 2012, A\&A, 540, A47

Fumagalli, M., Krumholz, M. R., Prochaska, J. X., Gavazzi, G., \& Boselli, A. 2009, ApJ, 697, 1811

Haynes, M. P., \& Giovanelli, R. 1984, AJ, 89, 758

Helou, G., Khan, I. R., Malek, L., \& Boehmer, L. 1988, ApJS, 68, 151

Hickson, P. 1982, ApJ, 255, 382

Hickson, P., Menon, T. K., Palumbo, G. G. C., \& Persic, M. 1989, ApJ, 341, 679

Hickson, P., Mendes de Oliveira, C., Huchra, J. P., \& Palumbo, G. G. 1992, ApJ, 399,353

Iglesias-Páramo, J., \& Vílchez, J. M. 1999, ApJ, 518, 94 
V. Martinez-Badenes et al.: Molecular gas content and SFR in Hickson compact groups

Jarrett, T. H., Chester, T., Cutri, R., et al. 2000, AJ, 119, 2498

Karachentseva, V. E. 1973, Soobshcheniya Spetsial'noj Astrofizicheskoj Observatorii, 8, 3

Kenney, J. D., \& Young, J. S. 1986, ApJ, 301, L13

Kennicutt, Jr., R. C. 1998, ARA\&A, 36, 189

Kroupa, P. 2001, MNRAS, 322, 231

Lavalley, M. P., Isobe, T., \& Feigelson, E. D. 1992, BAAS, 24, 839

Leon, S., Combes, F., \& Menon, T. K. 1998, A\&A, 330, 37

Leon, S., Verdes-Montenegro, L., Sabater, J., et al. 2008, A\&A, 485, 475

Leroy, A. K., Walter, F., Brinks, E., et al. 2008, AJ, 136, 2782

Leroy, A. K., Walter, F., Bigiel, F., et al. 2009, AJ, 137, 4670

Lisenfeld, U., Verdes-Montenegro, L., Sulentic, J., et al. 2007, A\&A, 462, 507

Lisenfeld, U., Espada, D., Verdes-Montenegro, L., et al. 2011, A\&A, 534, A102

Maloney, P., \& Black, J. H. 1988, ApJ, 325, 389

Menon, T. K. 1995, MNRAS, 274, 845

Narayanan, D., Krumholz, M., Ostriker, E. C., \& Hernquist, L. 2011, MNRAS, 418,664
Nishiyama, K., Nakai, N., \& Kuno, N. 2001, PASJ, 53, 757

Rasmussen, J., Ponman, T. J., Verdes-Montenegro, L., Yun, M. S., \& Borthakur, S. 2008, MNRAS, 388, 1245

Regan, M. W., Thornley, M. D., Helfer, T. T., et al. 2001, ApJ, 561, 218

Rubin, V. C., Hunter, D. A., \& Ford, Jr., W. K. 1990, ApJ, 365, 86

Sulentic, J. W., \& de Mello Rabaca, D. F. 1993, ApJ, 410, 520

Tzanavaris, P., Hornschemeier, A. E., Gallagher, S. C., et al. 2010, ApJ, 716, 556 Verdes-Montenegro, L., Yun, M. S., Perea, J., del Olmo, A., \& Ho, P. T. P. 1998, ApJ, 497, 89

Verdes-Montenegro, L., Yun, M. S., Williams, B. A., et al. 2001, A\&A, 377, 812 Verdes-Montenegro, L., Sulentic, J., Lisenfeld, U., et al. 2005, A\&A, 436, 443

Verdes-Montenegro, L., Yun, M. S., Borthakur, S., et al. 2007, in Groups of Galaxies in the Nearby Universe, ed. I. Saviane, V. D. Ivanov, \& J. Borissova, 349

Xu, C., \& Sulentic, J. W. 1991, ApJ, 374, 407

Young, J. S., \& Scoville, N. Z. 1991, ARA\&A, 29, 581 